\title{
Asymmetric and Symmetric Dimethylarginine as Risk Markers for Total Mortality and Cardiovascular Outcomes: A Systematic Review and Meta-Analysis of Prospective Studies
}

\author{
Sabrina Schlesinger ${ }^{1}$, Svenja R. Sonntag ${ }^{1}$, Wolfgang Lieb ${ }^{1 \oplus}$, Renke Maas ${ }^{2 \odot *}$ \\ 1 Institute of Epidemiology, Christian-Albrechts University of Kiel, Kiel, Germany, 2 Institute of Experimental \\ and Clinical Pharmacology and Toxicology, Friedrich-Alexander-Universität Erlangen-Nürnberg, Erlangen, \\ Germany \\ ه These authors contributed equally to this work. \\ * sabrina.schlesinger@epi.uni-kiel.de (SS); Renke.Maas@pharmakologie.uni-erlangen.de (RM)
}

\section{G open Access}

Citation: Schlesinger S, Sonntag SR, Lieb W, Maas $\mathrm{R}$ (2016) Asymmetric and Symmetric Dimethylarginine as Risk Markers for Total Mortality and Cardiovascular Outcomes: A Systematic Review and Meta-Analysis of Prospective Studies. PLoS ONE 11(11): e0165811. doi:10.1371/journal.pone.0165811

Editor: Tatsuo Shimosawa, The University of Tokyo, JAPAN

Received: May 31, 2016

Accepted: October 18, 2016

Published: November 3, 2016

Copyright: ๑ 2016 Schlesinger et al. This is an open access article distributed under the terms of the Creative Commons Attribution License, which permits unrestricted use, distribution, and reproduction in any medium, provided the original author and source are credited.

Data Availability Statement: All relevant data are within the paper and its Supporting Information files.

Funding: This work was supported by a grant from the German Research Foundation (DFG Excellence Cluster Inflammation at Interfaces EXC306/2).

Competing Interests: The author RM was named as a co-inventor on the patent "Method for determination of arginine, methylated arginines and derivatives thereof." EP1666884 A1/B1, US

\section{Abstract}

\section{Background}

A growing number of studies linked elevated concentrations of circulating asymmetric (ADMA) and symmetric (SDMA) dimethylarginine to mortality and cardiovascular disease (CVD) events. To summarize the evidence, we conducted a systematic review and quantified associations of ADMA and SDMA with the risks of all-cause mortality and incident CVD in meta-analyses accounting for different populations and methodological approaches of the studies.

\section{Methods}

Relevant studies were identified in PubMed until February 2015. We used random effect models to obtain summary relative risks (RR) and $95 \%$ confidence intervals $(95 \% \mathrm{Cls})$, comparing top versus bottom tertiles. Dose-response relations were assessed by restricted cubic spline regression models and potential non-linearity was evaluated using a likelihood ratio test. Heterogeneity between subgroups was assessed by meta-regression analysis.

\section{Results}

For ADMA, 34 studies (total $n=32,428$ ) investigating associations with all-cause mortality (events $=5,035$ ) and 30 studies (total $n=30,624$ ) investigating the association with incident CVD (events $=3,396$ ) were included. The summary RRs $(95 \% \mathrm{Cl}$ ) for all-cause mortality were 1.52 (1.37-1.68) and for CVD 1.33 (1.22-1.45), comparing high versus low ADMA concentrations. Slight differences were observed across study populations and methodological approaches, with the strongest association of ADMA being reported with all-cause mortality in critically ill patients. For SDMA, 17 studies (total $n=18,163$ ) were included for 
2006094122, DE602004020360 D1. The patent was owned and later transferred to a third party by his previous employer. The author does not work for the previous or current owner of the patent and receives no royalties or consulting fees from it and does not pursue any activity in relation to the patent.

\section{Abbreviations: ADMA, Asymmetric} dimethylarginine; AGXT2, alanine - glyoxylate aminotransferase 2; CVD, Cardiovascular disease; DDAH, dimethylarginine dimethylaminohydrolase; ELISA, Enzyme Linked Immunosorbent Assay; eNOS, endothelial nitric-oxide synthase; $F$, female; HDL, high density lipoprotein; HPLC, Highperformance liquid chromatography; HR, Hazard ratio; iNOS, inducible nitric-oxide synthase; $M$, male; MACE, major adverse cardiac event; NF-KB, nuclear factor kappa-light-chain-enhancer of activated B cells; nNOS, neuronal nitric oxide synthase; NO, Nitric oxide; OR, Odds ratio; PRMT, protein arginine $\mathrm{N}$-methyltransferase; ROS, reactive oxygen species; $R R$, Relative risk; $S D$, Standard deviation; SDMA, Symmetric dimethylarginine. all-cause mortality (events $=2,903$ ), and 13 studies (total $n=16,807$ ) for CVD (events = $1,534)$. High vs. low levels of SDMA, were associated with increased risk of all-cause mortality [summary RR $(95 \% \mathrm{Cl}): 1.31(1.18-1.46)$ ] and CVD [summary RR $(95 \% \mathrm{Cl}): 1.36$ (1.10-1.68) Strongest associations were observed in general population samples.

\section{Conclusions}

The dimethylarginines ADMA and SDMA are independent risk markers for all-cause mortality and CVD across different populations and methodological approaches.

\section{Introduction}

Asymmetric (ADMA) and symmetric (SDMA) dimethylarginine are both di-methylargingines that are structurally related to L-Arginine and, therefore, may interfere with L-Argininerelated signaling. Both share transport mechanisms of L-Arginine,[1] but likely differ in their functional effects on nitric oxide (NO) synthesis. Whereas ADMA is an established competitive inhibitor of the NO Synthasis, [2] SDMA has no or only little effect on NO-synthesis.[3] Particularly for ADMA, experimental and clinical data support a role in vascular remodeling, [4] [5] e.g. by demonstrating that vascular function and the degree of atherosclerosis correlated with ADMA levels in animal models.[5] On a parallel note, administration of ADMA in humans lead to reduced cardiac output and renal plasma flow and to increased vascular resistance.[6] Due to their biological functions, both markers have been explored as cardiovascular biomarkers.

Since the landmark studies by Zocalli et al.[7] and Valokonen et al.[8] in 2001, multiple studies have linked circulating ADMA concentrations to cardiovascular disease (CVD) risk and mortality and many reported positive associations.[9-11] However some conflicting results were observed; for example in the Framingham Offspring Study, ADMA was positively related to all-cause mortality, but not to incident CVD.[12] With respect to SDMA, initial studies did not find an association with adverse outcomes, $[7,13,14]$ but some more recent studies reported positive associations with all-cause mortality or CVD.[11, 15, 16] Overall, prior studies on ADMA/SDMA and CVD or all-cause mortality were derived from many different study populations (general population, $[15,17,18]$ patients with CVD, $[9,11,19,20]$ renal diseases, $[7$, $13,21-24]$ diabetes, $[25,26]$ or critically ill patients from intensive care unit,[27-29] respectively), differed in their methodological approaches (e.g. using types of samples (plasma vs. serum) or methods to determine the biomarker levels (HPLC vs. tandem mass spectroscopy vs. ELISA), or considered different confounders in their analysis.

A recent meta-analysis strengthened the notion that ADMA is an independent risk marker for cardiovascular events but could not confirm an association of SDMA with incident CVD. [30] While these data were intriguing, the exact shape of the dose-response relation between these dimethylarginines and CVD has not been described. In addition, the mentioned metaanalysis focused on ADMA/SDMA and CVD, but did not relate the biomarkers to all-cause mortality. Thus, we aim to quantify associations of ADMA and SDMA level with all-cause mortality and CVD in a systematic review and meta-analyses by accounting for differences in study populations (including participants from the general population as well as individuals with underlying diseases) and methodological approaches of the underlying studies. 


\section{Methods}

This report follows the Preferred Reporting Items for Systematic Reviews and Meta-Analyses (PRISMA) statement, [31] and the complete PRISMA checklist is provided in S1 Fig.

\section{Search strategy}

A systematic literature search was performed in MEDLINE (PubMed) by two independent investigators (S.S. and R.M.) to identify prospective studies, published until February 2015 that examined the relation between ADMA and SDMA (as exposure variables, each exposure considered separately) and all-cause mortality and CVD (as outcome, each outcome considered separately). There were no limits used in the searches.

Search terms included (ADMA OR dimethylarginin* OR SDMA) AND ("myocardial infarction" OR death OR mortality OR stroke OR "major adverse cardiac events" OR cardiovascular OR CAD OR "Coronary artery disease" OR CVD OR "Cardiovascular disease" OR "coronary heart disease" OR CHD) AND (Follow-up OR "clinical trial" OR Longitudinal OR prospective OR nested OR cohort OR observational OR endpoint OR "cox regression" OR outcome OR survival OR predict*). Furthermore, reference lists of relevant papers and previous reviews were hand-searched to assess additional potentially relevant articles.

\section{Data selection and extraction}

Inclusion criteria. We included studies if 1) studies had investigated the association between ADMA and/or SDMA with risk of all-cause mortality and/or CVD, 2) studies were original articles, 3 ) studies had a prospective design, 4) the associations was presented as odds ratio (OR), relative risk (RR) or hazard ratio (HR). In the present manuscript RR stands for all three estimates.

Studies were excluded if 1) they were not reported in English language, 2) they focused on a mixed endpoint (combined endpoint e. g. major adverse cardiac event (MACE), including allcause mortality and CVD), and 3) studies did not report how ADMA and SDMA were modeled in the statistical analyses (e. g. if it was not clear whether they were modeled as categorical or continuous traits; or when it was unclear whether effect estimates were given per 1-unit or per 1-SD increment in biomarker levels). If such relevant information was missing in the publication, we contacted the corresponding author, and if authors responded, studies were included in our meta-analysis.[10, 16, 29, 32-34]

In the case that multiple studies reported on one dataset, we included the most recent study in our meta-analysis.

Furthermore, three investigators (S.S., R.M. and S.R.S.) independently extracted for each study, information about the first author, publication year, country of the study, study source and design, duration of follow-up, characteristics of the study sample (general population or patients with specific diseases), sex, age, sample size, outcome, type of estimate, number of cases, exposure assessment and categorization, as well as the most comprehensively adjusted OR, RR or HR, with the respective adjustment variables. Estimates and their $95 \%$ confidence intervals (95\% CI) for ADMA/SDMA in relation to all-cause mortality or CVD were extracted as they were presented in the original reports, including estimates per 1-unit increments, per 1-SD increment, or for the highest versus the lowest categories (e.g. tertiles or quartiles) of ADMA or SDMA, respectively. We contacted the corresponding author, if relevant information was missing.

Definition of outcomes. We focused on two outcomes: 1) all-cause mortality and 2) CVD, which was defined as fatal and nonfatal CVD and coronary heart disease events, as it has been done in previous work. [35] Studies that provided effect estimates for a combined 
outcome, including all-cause mortality and CVD, were not included in our meta-analysis. If studies reported findings for defined sub-outcomes (e.g. cause-specific mortality) or subgroups of the overall sample (e.g. stratified for patients with and without surgery), we combined theses sub-group/sub-outcome results to estimate effect estimates for the overall sample, $[9,36-38]$ by using fixed effect models.

\section{Statistical analyses}

We conducted four main analyses: we investigated the associations between 1) ADMA levels and all-cause-mortality, 2) ADMA levels and incident CVD, 3) SDMA levels and all-cause mortality, and 4) SDMA levels and incident CVD.

High versus low biomarker level. In the individual studies the exposure variable (the biomarker concentrations) were modeled in different ways (either as a continuous or as a categorical trait) and the effect measures (HRs/RRs/ORs with their corresponding 95\% CIs) were reported per different increments in the exposure variable (e.g. per $0.1 \mu \mathrm{mol} / \mathrm{L}$, per $1 \mu \mathrm{mol} / \mathrm{L}$ or per $1-\mathrm{SD}$ increment in the continuous biomarker; or per tertiles, quartiles or quintiles in biomarker levels; or comparing individuals above vs. below the median). In the present meta-analysis, we harmonized the presentation of the data by providing effect measures for the top vs. the bottom tertile of the ADMA or SDMA distribution. If the effect measures in the original publication were not presented per tertiles, the results had to be converted to a standard scale of effect, by giving an estimate per 2.18 SD units of ADMA or SDMA as described by Danesh et al.[39] The factor of 2.18 is the difference in the means of the upper and lower tertile of the standard normal distribution. Thus, this scaling method assumes that the exposure (ADMA or SDMA) follows a normal distribution and the association with disease risk (CVD or mortality) is log-linear. If the original publication reported effect measures by biomarker quartiles, the effect measure of the top vs. the bottom quartile was log-transformed, then multiplied by the factor 2.18/2.54 and the resulting product was exponentiated to the base of e to derived a RR comparing the top vs. the bottom tertile.[39] For studies reporting results by biomarker quintiles or comparing individuals above vs. below the median we used scaling factors of 2.18/2.80 and 2.18/1.59, respectively.[39] If the original studies reported relative risk increases per 1-SD increment in ADMA or SDMA levels, the log risk ratio was multiplied by 2.18 and subsequently presented as effect measure of the top vs. bottom tertile. Risk ratio that were reported per 1-unit increment, were multiplied by the study specific SD. If some individual studies did not report the study specific biomarker SD for their sample, we used the SD of the largest study $(0.14 \mu \mathrm{mol} / \mathrm{L}$ for ADMA and $0.12 \mu \mathrm{mol} / \mathrm{L}$ for SDMA; Email communication with the corresponding author of that study).[16]

Summary RRs (95\% CIs) for ADMA and SDMA (each biomarker considered separately) and all-cause mortality and/or CVD (each outcome considered separately) were calculated by applying random effect models. Heterogeneity between studies was evaluated using $\mathrm{I}^{2}$ statistics.

Subgroup analyses. To assess for risk of bias, we conducted subgroup analyses considering the following factors:

- underlying study population (general population, patients with renal disease, patients with prevalent CVD, patients with diabetes mellitus, and, for the analyses with respect to all-cause mortality, critically ill patients from intensive care units),

- number of events $(<100,100-<200$ and $\geq 200)$,

- duration of follow-up (longer or shorter than the mean across all studies: $<4.7$ years and $\geq 4.7$ years),

- type of blood sample (plasma and serum) used for analyses, 
- method for ADMA/SDMA measurement (HPLC, tandem mass spectrometry and ELISA), and

- adjustment for number of important confounders $(0-2,3-5$ and $\geq 6$, whereas important confounders included age, sex, BMI/or waist circumference, smoking, history of CVD, diabetes, blood pressure/hypertension, blood lipids/hyperlipidemia, family history of CVD and GFR/ eGRF).

Heterogeneity between subgroups was assessed by applying meta-regression analysis.

Dose response meta-analysis. In a dose-response meta-analysis, we investigated ADMA and SDMA as continuous traits (effect measures per $0.1 \mu \mathrm{mol} / \mathrm{L}$ in biomarker levels) in relation to all-cause mortality and CVD, respectively. If the original publication provided estimates per $1 \mu \mathrm{mol} / \mathrm{L}$ increment in biomarker levels, these were converted into estimates per $0.1 \mu \mathrm{mol} / \mathrm{L}$ increment. If studies did not report the association with all-cause mortality, or CVD with ADMA or SDMA being modeled as a continuous trait but rather for categories of the biomarker, we used the method described by Orsini et al. and by Greenland and Longnecker.[40, 41] Here, the study specific slopes and 95\% CIs were estimated from the natural logarithms of the RRs and 95\% CIs across the categories of ADMA or SDMA, respectively. This requires from each individual study the RR (with 95\% CI), the quantified exposure value, and the number of cases and person years for at least three exposure categories.

For studies that did not report the number of person-years in the individual exposure categories, we estimated the person-years per category based on the number of cases per category and the total person-years/ total number of participants and the follow-up period of the entire study, as suggested previously.[42, 43]

For any missing information, we contacted the corresponding author.[15, 16, 44-46] If missing information could not be obtained, we excluded the respective studies from the doseresponse analysis. $[15,45,46]$ If the author reported the range (rather than the mean) to define an exposure category, we estimated the mean value as previously described.[47, 48]

Finally, we performed cubic spline regression models to explore a potential non-linear relation between di-methylarginines (ADMA/ SDMA) and all-cause mortality or CVD, respectively. For this specific analysis only studies with information on at least three exposure categories (please see above) could be included. To formally test for non-linearity, we used a likelihood ratio test.[49] In a sensitivity analysis, we restricted our analysis to studies which determined ADMA/SDMA by tandem mass spectrometry and ELISA because these methods allowed more reliable quantification of the absolute concentration as compared to the assessment by some of the early HPLC methods (predominantly used in older studies).

All analyses were performed using SAS version 9.3 (SAS Institute, Inc., Cary, NC, USA), R statistical software version 3.0.3 (R Foundation for Statistical Computing, Vienna, Austria) and the R package meta version 3.6-0 (Schwarzer, Freiburg, Germany) and metafor version 1.9-3 (Viechtbauer, Maastricht, The Netherlands). A two-tailed p-value of $<0.05$ was considered as statistical significant.

\section{Results}

\section{Literature search and study characteristics}

Overall, we identified 711 studies, from which titles and abstracts were reviewed. After screening full-texts of 102 studies, we finally included 50 studies in our meta-analysis (S2 Fig).

Out of these, 34 studies investigated the association between ADMA and all-cause mortality, 30 studies evaluated the association between ADMA and CVD, and 17 studies examined the relation between SDMA and all-cause. A total of 13 studies assessed the association of 
SDMA with CVD. Across all studies, the mean follow-up time was 4.7 years (range: $0.1-11.3$ years).

Characteristics of the included studies are provided in Table 1 and S1 Table. A total of 8 studies were based on participants of the general population, 12 studies included patients with renal disease, 23 studies included individuals with prevalent CVD, 4 studies provided results for individuals with diabetes mellitus, and 4 studies included patients from the intensive care unit. ADMA and SDMA were measured in plasma in 35 and in serum in 15 studies. In addition, ADMA and SDMA were analyzed using HPLC in 27 studies, using tandem mass spectrometry in 13 studies and with ELISA in 10 studies. The confounders included in the individual studies are shown in the S1 Table.

\section{Association between ADMA and all-cause mortality}

Across all studies ( $\mathrm{n}=34$, involving 32,428 subjects of which 5,035 died; mean follow-up: 5.4 years), higher ADMA levels were associated with greater risk for all-cause mortality (summary RR: 1.52; 95\% CI, 1.37-1.68, $\mathrm{p}<0.0001$; comparing participants in the top ADMA tertile with participants in the bottom ADMA tertile; Fig 1a). There was evidence for statistical significant heterogeneity across studies $\left(\mathrm{I}^{2}=88.9 \%, \mathrm{p}<0.0001\right)$.

In subgroup analyses, the strongest association was reported in critically ill patients from intensive care units [summary RR: 4.85; 95\% CI, 1.39-16.97, $\mathrm{p}=0.014$; based on 4 studies ( pheterogenity $<0.001$ ); Table 2]. However, the association between ADMA and all-cause mortality was also statically significant in the general population, and in patients with renal diseases and in those with prevalent CVD. The association in patients with diabetes mellitus reached borderline significance [summary RR: 1.49; 95\% CI, 0.99-2.25, $\mathrm{p}=0.068$; based on 4 studies]. In addition, the magnitude of the reported effect tended to be greater in smaller samples and decreased in samples with a larger number of cases $\left(p_{\text {heterogenity }}<0.0001\right)$ and with a longer duration of follow-up ( $p_{\text {heterogenity }}=0.001$ ). The effect was slightly stronger in studies measuring ADMA in plasma as compared to studies measuring ADMA in serum ( $\mathrm{p}_{\text {heterogenity }}=$ 0.033). As expected, studies with more comprehensive adjustments displayed weaker associations than studies including fewer confounders ( $\mathrm{p}_{\text {heterogenity }}=0.002$ ).

The dose-response meta-analysis revealed that the relative risk for all-cause mortality increased by 7\% per each $0.1 \mu \mathrm{mol} / \mathrm{L}$ increment of ADMA [summary RR: 1.07, 95\% CI, 1.05$1.10, \mathrm{p}<0.0001$; based on 15 studies]. However, there was evidence for a non-linear association between ADMA and all-cause mortality ( $\mathrm{p}$ for nonlinearity $=0.035$, based on 7 studies; Fig $1 \mathrm{~b}$ ). In a sensitivity analysis, excluding older studies which determined ADMA by HPLC, there was no indication for a non-linear relation ( $\mathrm{p}$ for nonlinearity $=0.184$, based on 3 studies).

\section{Association between ADMA and CVD}

We observed a positive association of ADMA levels with incident CVD, based on 30 studies with 30,624 subjects and 3,396 incident CVD events; mean follow-up: 6.0 years). Individuals in the top ADMA tertile had 33\% greater risk of CVD as compared to individuals in the bottom tertile (summary RR; 1.33 ; 95\% CI, 1.22-1.45, p $<0.0001$; Fig $2 \mathrm{a}$ ). Statistically significant heterogeneity across studies was observed $\left(I^{2}=76.6 \%, \mathrm{p}<0.0001\right)$.

In stratified analyses, the positive association between ADMA and CVD could be observed in all relevant subgroups, e.g. in the general population and all clinical samples, in larger and smaller studies, and in studies with longer and shorter follow-up periods (Table 2).

Modeled as a continuous trait, we observed a 5\% increase in CVD risk per each $0.1 \mu \mathrm{mol} / \mathrm{L}$ increment in ADMA [summary RR (95\% CI): 1.05 (1.03-1.06), p<0.0001; including 19 studies]. There was no evidence for a non-linear relation between ADMA and CVD ( $p$ for non- 
1)

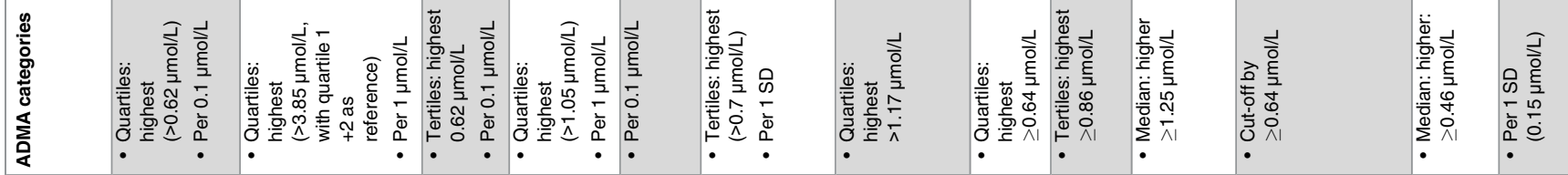

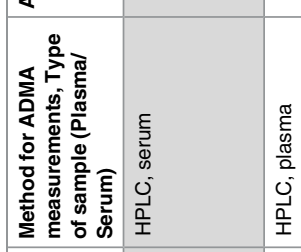

ำ

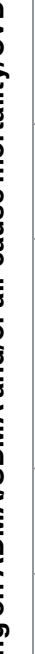

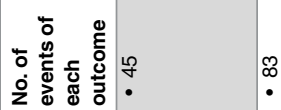
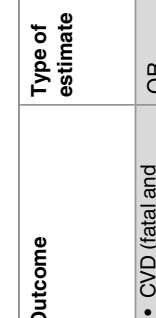

0

密

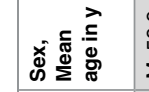

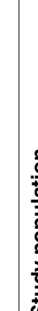

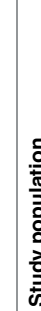

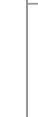

흘

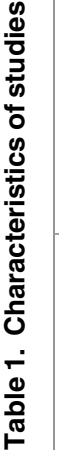

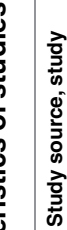

(a)

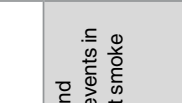
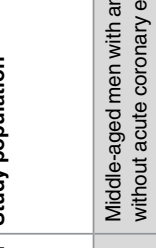

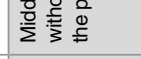
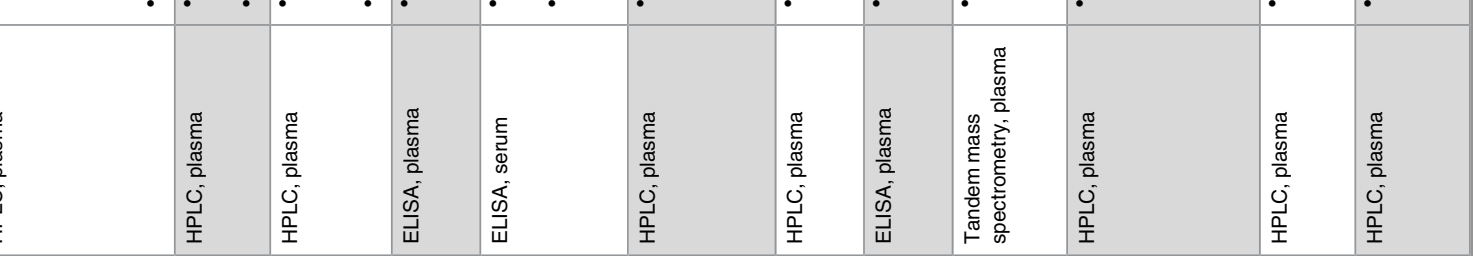

$\infty$

ก.

হ.

\&

$\begin{array}{llll} \pm & \infty & \bar{m} \\ \bullet & \stackrel{0}{*} & 0\end{array}$

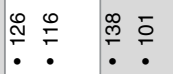

\begin{tabular}{|c|c|c|c|c|}
\hline 또 & $\underset{\mathbb{x}}{\mathbb{\alpha}}$ & ఝ̊ & $\underline{\underline{x}}$ & $\stackrel{\dddot{x}}{\underline{x}}$ \\
\hline 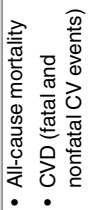 & 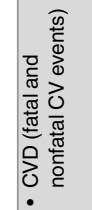 & 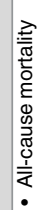 & 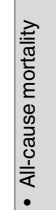 & 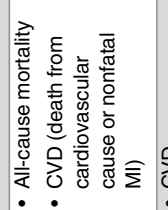 \\
\hline
\end{tabular}

采

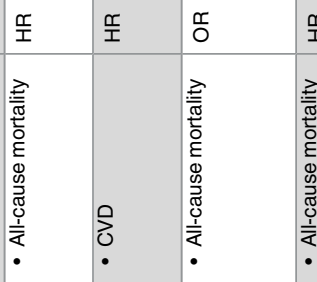

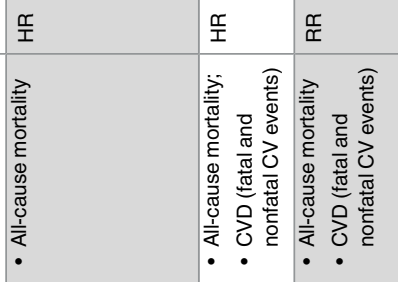

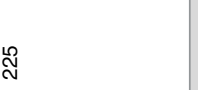

埠

$\stackrel{\frac{0}{\infty}}{\stackrel{0}{2}}$

每

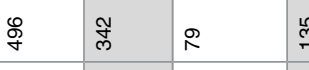

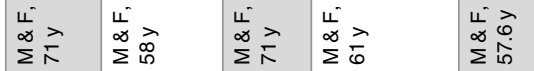

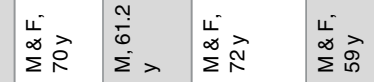

弇

崖

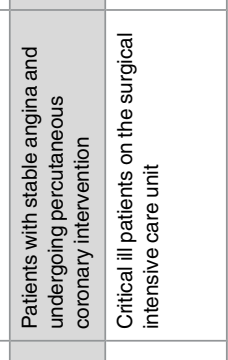

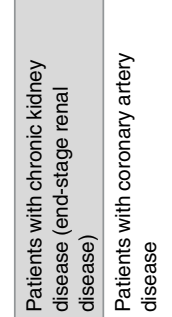

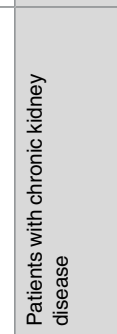

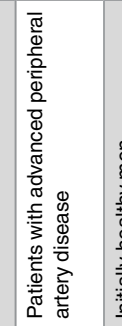

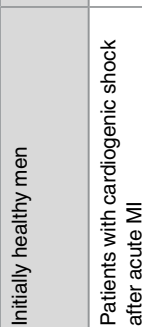

\begin{tabular}{|c|}
\hline 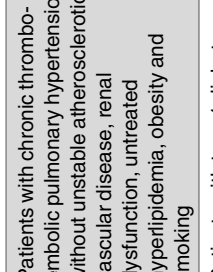 \\
\hline
\end{tabular}

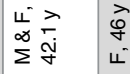

\begin{tabular}{|c|c|c|c|c|c|c|c|c|c|c|c|c|}
\hline$\vec{\wedge}$ & 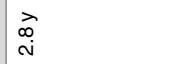 & 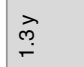 & ๘ & ले & 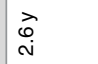 & $\overrightarrow{\mathrm{N}}$ & $\stackrel{\vec{o}}{\stackrel{\leftrightarrow}{\prime}}$ & 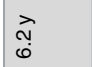 & $\underset{0}{\overrightarrow{0}}$ & $\frac{\vec{\infty}}{\vec{\infty}}$ & $\stackrel{\vec{m}}{\stackrel{\vec{p}}{F}}$ & $\vec{d}$ \\
\hline 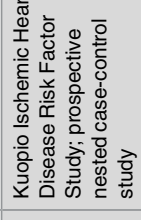 & 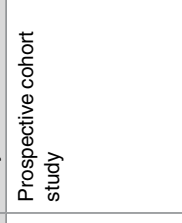 & 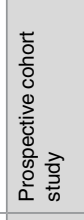 & 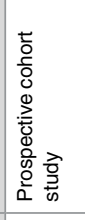 & 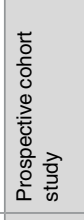 & 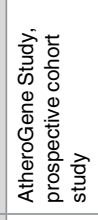 & 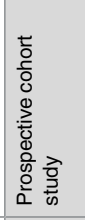 & 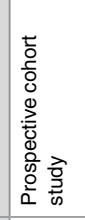 & 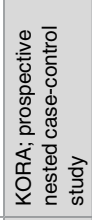 & 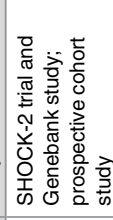 & 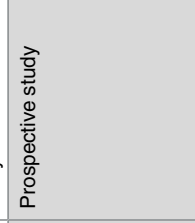 & 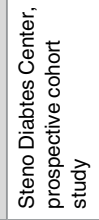 & 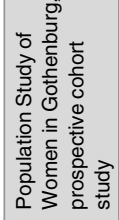 \\
\hline 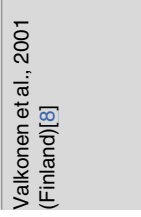 & 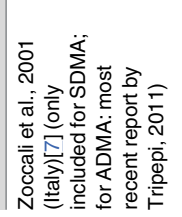 & 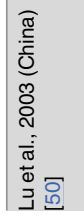 & 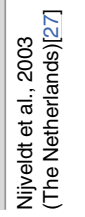 & 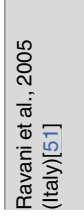 & 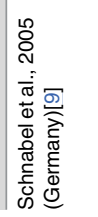 & 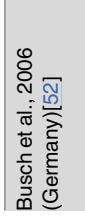 & 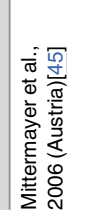 & 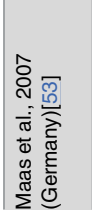 & 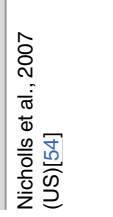 & 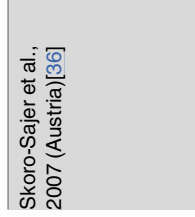 & 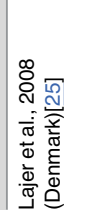 & 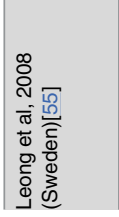 \\
\hline
\end{tabular}




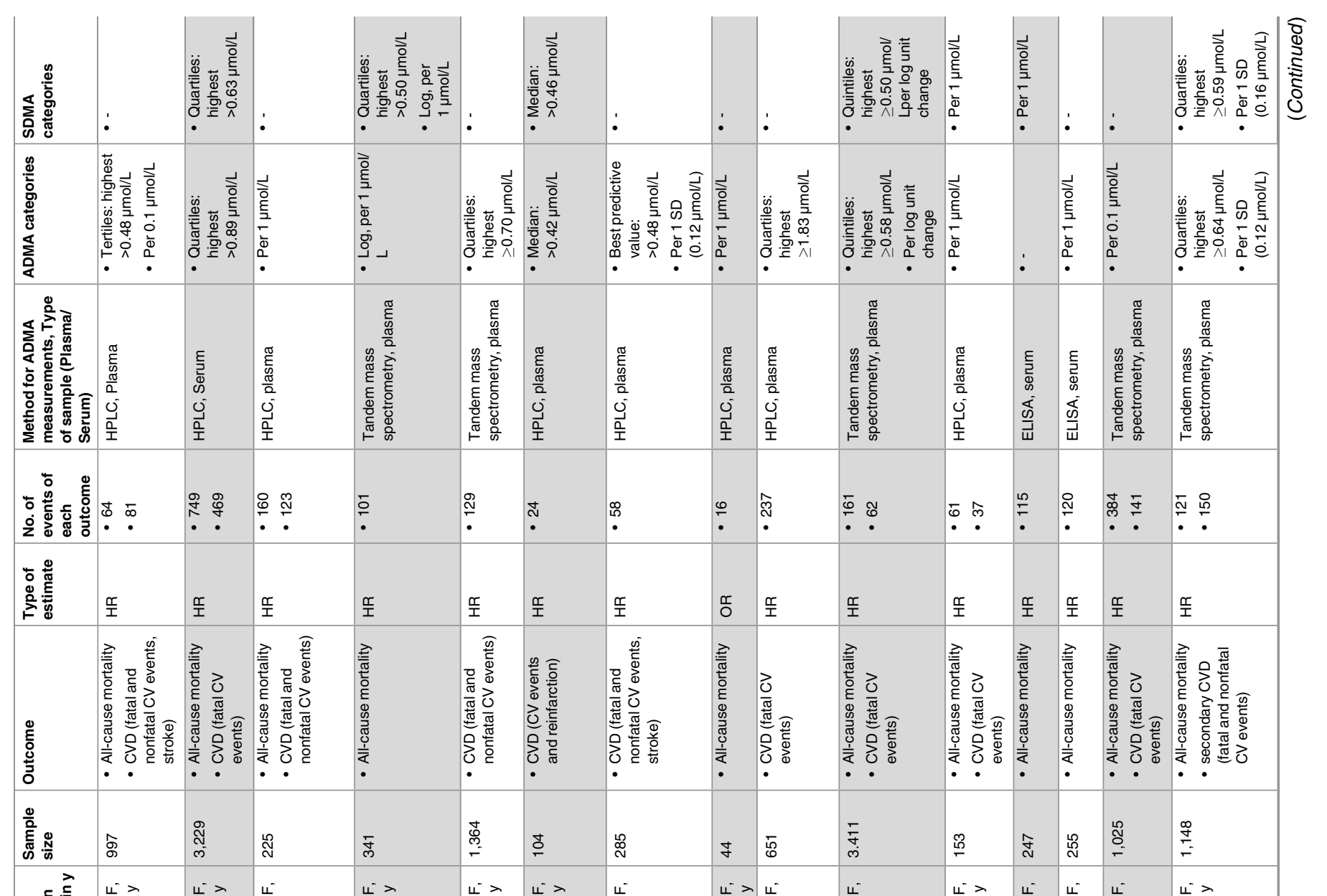

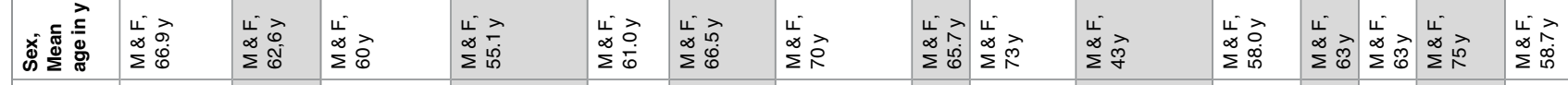

\begin{tabular}{|c|c|c|c|c|c|c|c|c|c|c|c|c|c|c|}
\hline 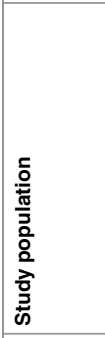 & 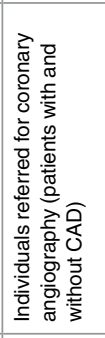 & 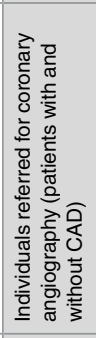 & 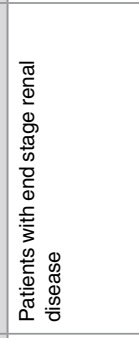 & 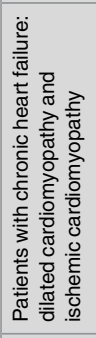 & 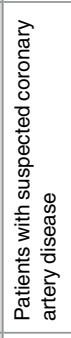 & 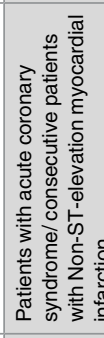 & 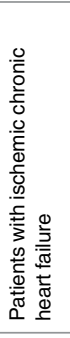 & 它 & 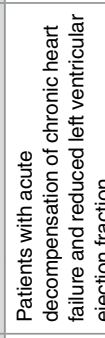 & & 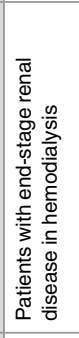 & 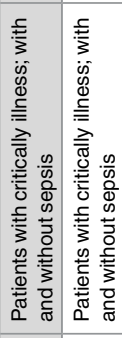 & 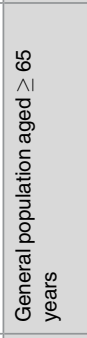 & 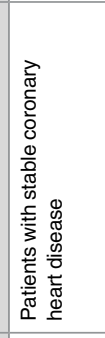 \\
\hline 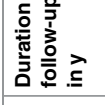 & 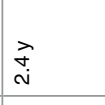 & $\stackrel{\lambda}{i}$ & $\stackrel{\vec{p}}{\underline{m}}$ & ले & 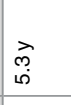 & $\stackrel{\vec{\infty}}{=}$ & \े & $\cong$ & $\geq$ & $\stackrel{\vec{T}}{i}$ & $\overrightarrow{~ ल े ~}$ & के गे & సे & $\vec{\infty}$ \\
\hline 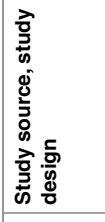 & 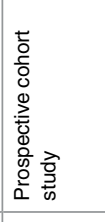 & 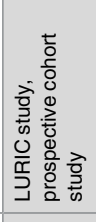 & 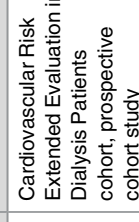 & 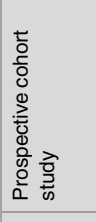 & 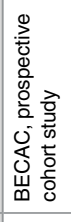 & $\begin{array}{l}\frac{0}{0} \\
\frac{0}{2} \\
\frac{2}{2}\end{array}$ & 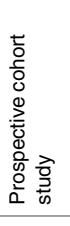 & 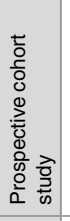 & 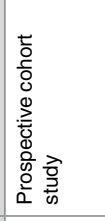 & 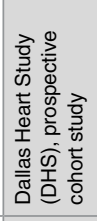 & 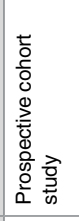 & 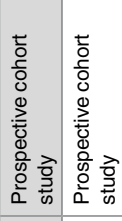 & 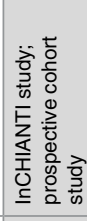 & 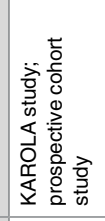 \\
\hline 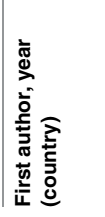 & 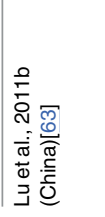 & 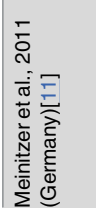 & 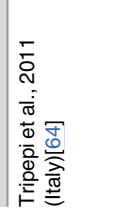 & 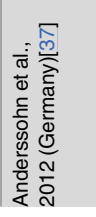 & 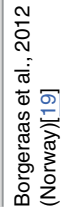 & 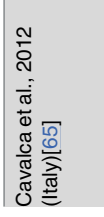 & 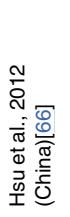 & 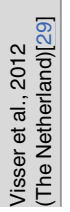 & 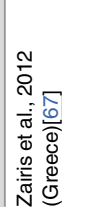 & 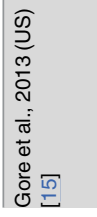 & 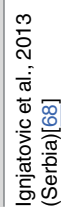 & 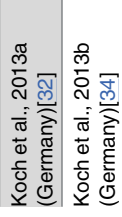 & 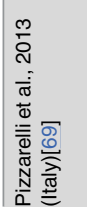 & 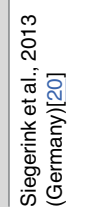 \\
\hline
\end{tabular}




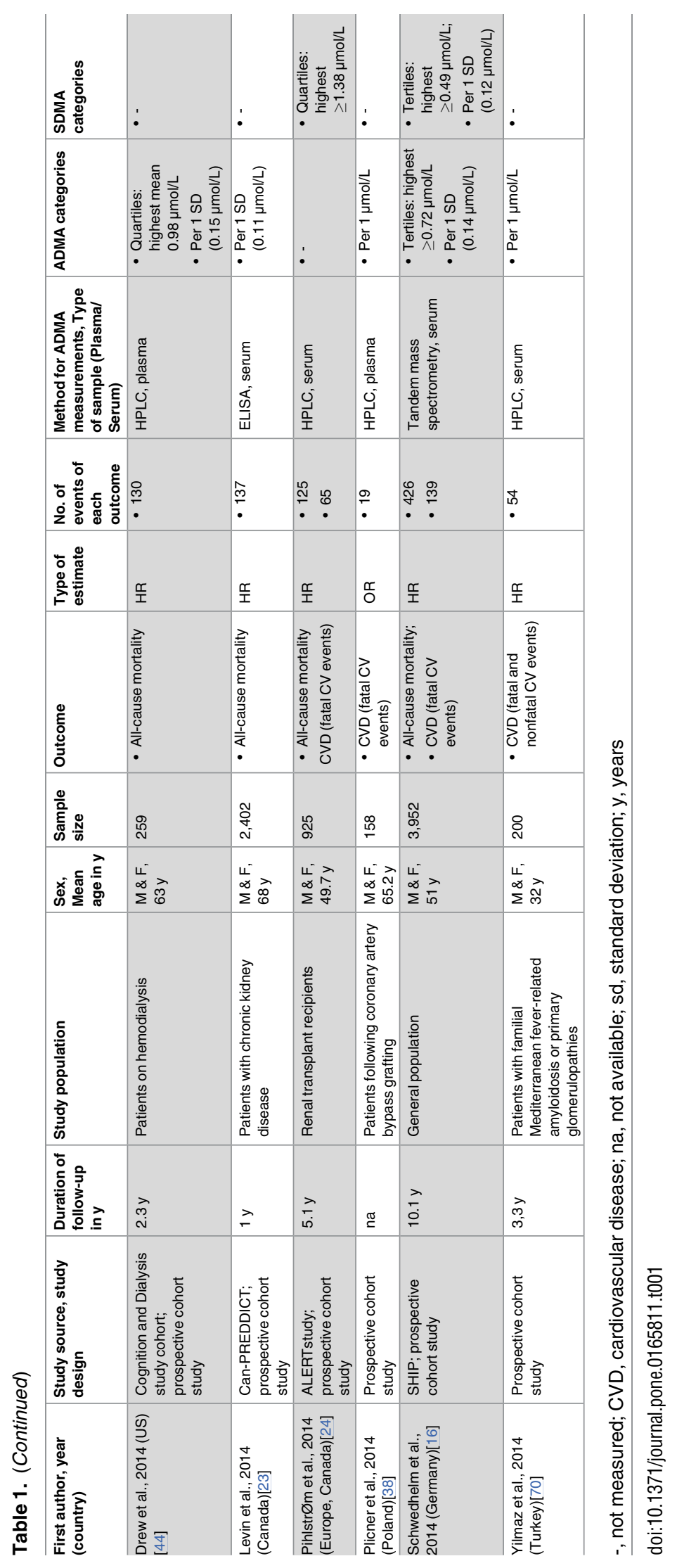


a) Study

Nijveldt, 2003 Ravani, 2005 Schnabel, 2005 Mittermayer, 2006 Nicholls, 2007 Skoro-Sajer, 2007 Lajer, 2008 Leong, 2008 Wilson Tang, 2008 Zeller, 2008 Aucella, 2009 Böger, 2009 Cavusoglu, 2009 Wang, 2009 Young, 2009 Abedini, 2010 Cavusoglu, 2010 Schulze, 2010 Yeo, 2010 Böger, 2011 Davis, 2011 Lu, 2011 Meinitzer, 2011 Tripepi, 2011 Anderssohn, 2012 Visser, 2012 Gore, 2013 Ignjatovic, 2013 Koch, 2013b Pizzarelli, 2013 Siegerink, 2013 Drew, 2014 Levin, 2014

Schwedhelm, 2014

Random effects mode Heterogeneity: $p<0.0001$
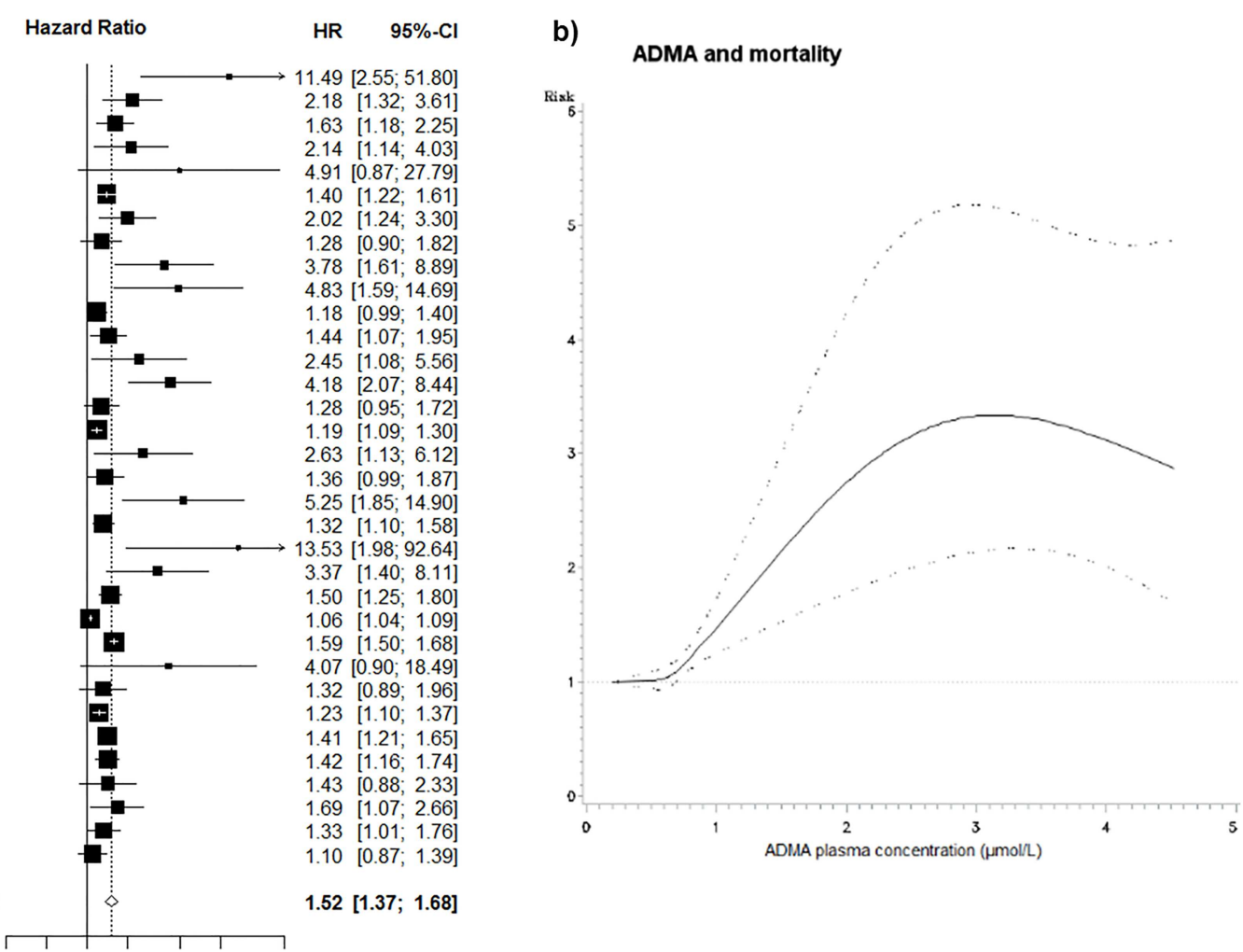

Fig 1. ADMA and all-cause mortality: a) high versus low analysis, and b) non-linear dose-response analysis (based on 7 studies, $\mathbf{p}$ for non-linearity $\mathbf{0} \mathbf{0 . 0 3 5}$ ). Black square: point estimate for individual study; horizontal line: $95 \% \mathrm{Cl}$ for observed effect in each study; diamond: pooled estimate and $95 \% \mathrm{Cl}$ for meta-analysis. Random-effects estimate (DerSimonian and Laird method).

doi:10.1371/journal.pone.0165811.g001

linearity $=0.370$, based on 10 studies; Fig 2b). Excluding studies which determined ADMA with HPLC, results remained unchanged ( $\mathrm{p}$ for non-linearity $=0.992$, based on 10 studies).

\section{Association between SDMA and all-cause mortality}

In our meta-analysis, based on 17 studies and (18,163 individuals, 2,903 deaths; mean followup 4.6 years) SDMA was associated with increased risk of all-cause mortality in the top as compared to the bottom SDMA tertile [(summary RR (95\% CI): 1.31 (1.18-1.46), $\mathrm{p}<0.0001$; Fig 3a]. There was evidence for statistically significant heterogeneity between studies $\left(\mathrm{I}^{2}=\right.$ $82.1 \%, \mathrm{p}<0.0001$ ). In subgroups analyses (Table 3 ), the association was stronger in the general population as compared to defined clinical settings, ( $p_{\text {heterogeneity }}=0.003$ across all samples), and more prominent in studies with a larger number of events ( $p_{\text {heterogeneity }}=0.009$ ) and longer duration of follow-up ( $\mathrm{p}_{\text {heterogeneity }}=0.004$ ). We also observed slight differences in the strength (but not in the direction) of the association depending on the method of SDMA assessment ( pheterogeneity $=0.020$ ). As expected, studies with a parsimonious set of confounders reported stronger associations as compared to full-adjusted analyses $\left(p_{\text {heterogeneity }}=0.055\right)$.

The dose-response meta-analysis for all-cause mortality revealed in a $4 \%$ risk increase ( $95 \%$ CI: $2 \%-5 \%$ ) per $0.1 \mu \mathrm{mol} / \mathrm{L}$ increment of SDMA ( $<<0.0001$, based on 10 studies). However, there was also an indication for a non-linear association between SDMA and all-cause mortality ( $\mathrm{p}$ for non-linearity $=0.010$, based on 4 studies; Fig $3 \mathrm{~b}$ ). When excluding studies HPLC- 
Table 2. Meta-Analysis of ADMA and all-cause mortality or CVD for subgroups.

\begin{tabular}{|c|c|c|c|c|c|c|}
\hline Factors stratified & $\begin{array}{c}\text { summary RR } \\
(95 \% \mathrm{Cl}) \neq\end{array}$ & $\begin{array}{c}\text { No. of } \\
\text { studies }\end{array}$ & $\begin{array}{c}\text { P for heterogeneity between } \\
\text { subgroups } \dagger\end{array}$ & $\begin{array}{c}\text { summary RR } \\
(95 \% \mathrm{Cl}) \neq\end{array}$ & $\begin{array}{c}\text { No. of } \\
\text { studies }\end{array}$ & $\begin{array}{c}\text { P for heterogeneity between } \\
\text { subgroups } \dagger\end{array}$ \\
\hline & \multicolumn{3}{|c|}{ All-cause mortality } & \multicolumn{3}{|c|}{ CVD } \\
\hline All & $1.52(1.37-1.68)$ & 34 & & $1.33(1.22-1.45)$ & 30 & \\
\hline \multicolumn{7}{|l|}{ Study population } \\
\hline General population & $1.30(1.16-1.47)$ & 5 & \multirow[t]{5}{*}{0.001} & $1.34(1.12-1.60)$ & 8 & \multirow[t]{5}{*}{0.170} \\
\hline $\begin{array}{l}\text { Patients with renal } \\
\text { diseases }\end{array}$ & $1.22(1.11-1.35)$ & 8 & & $1.17(1.06-1.29)$ & 9 & \\
\hline $\begin{array}{l}\text { Patients with existing } \\
\text { CVD }\end{array}$ & $1.67(1.46-1.90)$ & 16 & & $1.49(1.30-1.72)$ & 14 & \\
\hline $\begin{array}{l}\text { Patients with } \\
\text { diabetes }\end{array}$ & $1.49(0.99-2.25)$ & 4 & & $1.94(0.80-4.66)$ & 2 & \\
\hline $\begin{array}{l}\text { Patients from } \\
\text { intensive care unit }\end{array}$ & $\begin{array}{c}4.85(1.39- \\
16.97)\end{array}$ & 4 & & - & & \\
\hline \multicolumn{7}{|l|}{ Number of events } \\
\hline$<100$ & $2.43(1.84-3.21)$ & 14 & \multirow[t]{3}{*}{$<0.0001$} & $1.35(1.19-1.53)$ & 16 & \multirow[t]{3}{*}{0.279} \\
\hline $100-<200$ & $1.46(1.23-1.74)$ & 12 & & $1.48(1.17-1.85)$ & 9 & \\
\hline$\geq 200$ & $1.29(1.20-1.40)$ & 8 & & $1.23(1.10-1.37)$ & 5 & \\
\hline \multicolumn{7}{|c|}{ Duration of follow-up (by mean)* } \\
\hline$<4.7$ years & $1.65(1.46-1.87)$ & 18 & \multirow[t]{2}{*}{0.001} & $1.37(1.18-1.59)$ & 13 & \multirow[t]{2}{*}{0.640} \\
\hline$\geq 4.7$ years & $1.27(1.16-1.40)$ & 13 & & $1.31(1.17-1.47)$ & 16 & \\
\hline \multicolumn{7}{|l|}{ Blood sample } \\
\hline Plasma & $1.64(1.42-1.88)$ & 24 & \multirow[t]{2}{*}{0.033} & $1.29(1.15-1.44)$ & 21 & \multirow[t]{2}{*}{0.466} \\
\hline Serum & $1.35(1.21-1.51)$ & 10 & & $1.37(1.22-1.54)$ & 10 & \\
\hline \multicolumn{7}{|l|}{ Method } \\
\hline HPLC & $1.45(1.27-1.65)$ & 16 & \multirow[t]{3}{*}{0.962} & $1.34(1.20-1.50)$ & 18 & \multirow[t]{3}{*}{0.457} \\
\hline $\begin{array}{l}\text { Tandem mass } \\
\text { spectrometry }\end{array}$ & $1.47(1.28-1.69)$ & 11 & & $1.25(1.10-1.41)$ & 8 & \\
\hline ELISA & $1.49(1.30-1.72)$ & 7 & & $1.52(1.11-2.08)$ & 4 & \\
\hline \multicolumn{7}{|c|}{ Adjustment for important confounders } \\
\hline $0-2$ & $2.21(1.70-2.87)$ & 12 & \multirow[t]{3}{*}{0.002} & $1.25(1.07-1.46)$ & 6 & \multirow[t]{3}{*}{0.260} \\
\hline $3-5$ & $1.41(1.21-1.65)$ & 8 & & $1.60(1.24-2.06)$ & 7 & \\
\hline$\geq 6$ & $1.34(1.20-1.49)$ & 14 & & $1.31(1.17-1.46)$ & 17 & \\
\hline
\end{tabular}

$\mathrm{Cl}$, confidence interval; CVD, cardiovascular disease; HPLC, High-performance liquid chromatography; HR, hazard ratio; ELISA, Enzyme Linked Immunosorbent Assay.

${ }^{\ddagger}$ summary RRs are derived from the maximally adjusted models.

${ }^{\dagger} \mathrm{P}$ for heterogeneity between subgroups was evaluated by meta-regression analysis.

* for all-cause mortality for three studies and for CVD for one study time of follow-up was not available.

doi:10.1371/journal.pone.0165811.t002

based measurements, there was no evidence for non-linearity $(\mathrm{p}=0.425)$. However, this analysis was based on 2 studies only.

\section{Association between SDMA and CVD}

The risk for incident CVD (based on 13 studies, including 16,807 subjects and 1,534 cases; mean follow-up 4.9 years) was $36 \%$ higher for participants in the upper tertile compared to participants in the lowest tertile [summary RR (95\% CI): 1.36 (1.10-1.68); Fig 4a). Statistically significant heterogeneity between studies was observed $\left(\mathrm{I}^{2}=76.6 \% ; \mathrm{p}<0.0001\right)$. In subgroup 
a) Study

Valkonen, 2001
Lu, 2003
Schnabel, 2005
Busch, 2006
Maas, 2007
Lajer, 2008
Leong, 2008
Zeller, 2008
Aucella, 2009
Böger, 2009
Kiechl, 2009
Young, 2009
Abedini, 2010
Ari, 2010
Shi, 2010
Böger, 2011
Lu, 2011
Meinitzer, 20111
Tripepi, 2011
Borgeraas, 2012
Cavalac, 2012
Hsu, 2012
Zairis, 2012
Gore, 2013
Ignjatoviv, 2013
Pizzarelli, 2013
Siegrink, 2013
Plicner, 2014
Schwedhelm, 2014
Yilmaz, 2014

Yilmaz, 2014

Random effects model Heterogeneity: $p<0.0001$

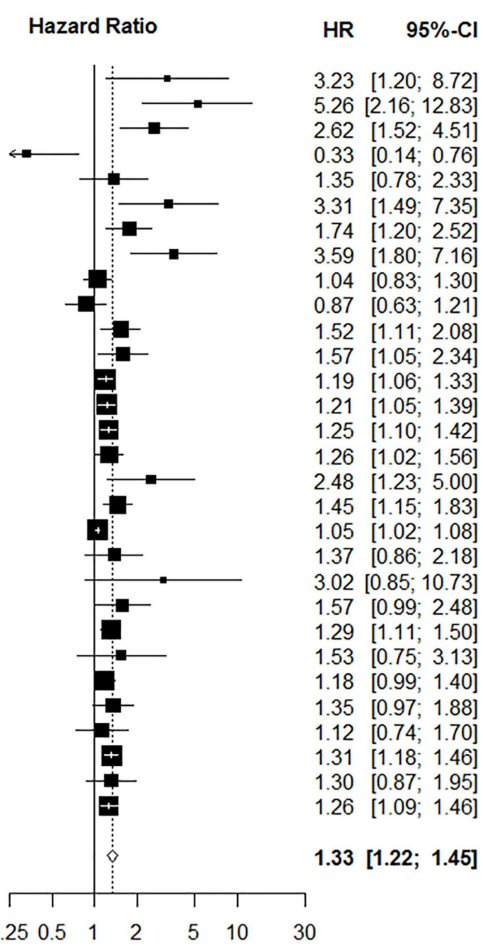

b)
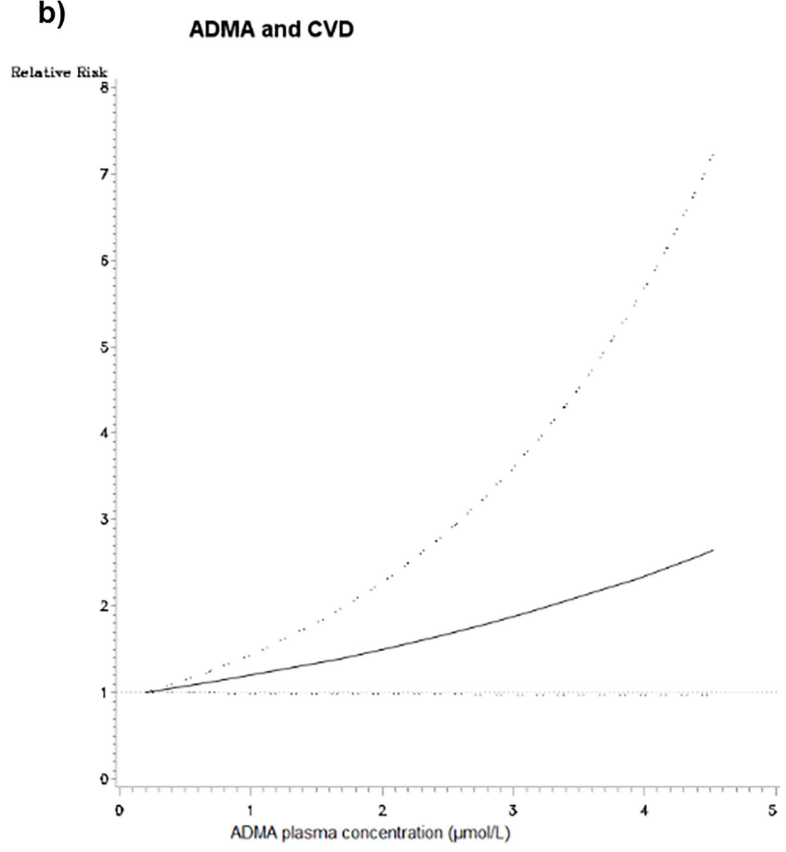

Fig 2. ADMA and CVD: a) high versus low analysis, and b) non-linear dose-response analysis (based on 10 studies, $p$ for nonlinearity = 0.370). Black square: point estimate for individual study; horizontal line: $95 \% \mathrm{Cl}$ for observed effect in each study; diamond: pooled estimate and $95 \% \mathrm{Cl}$ for meta-analysis. Random-effects estimate (DerSimonian and Laird method).

doi:10.1371/journal.pone.0165811.g002

a)

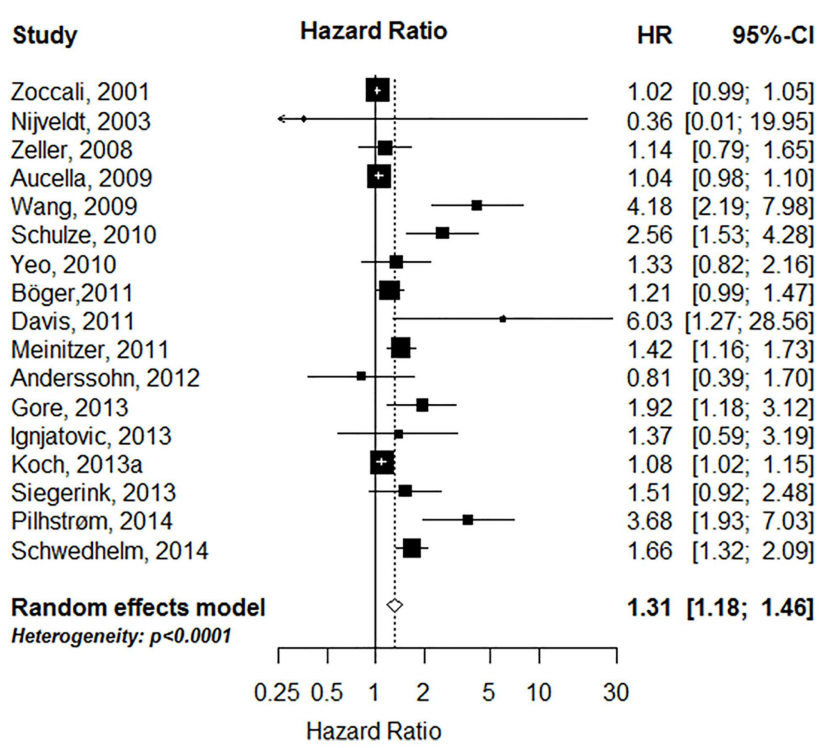

b)

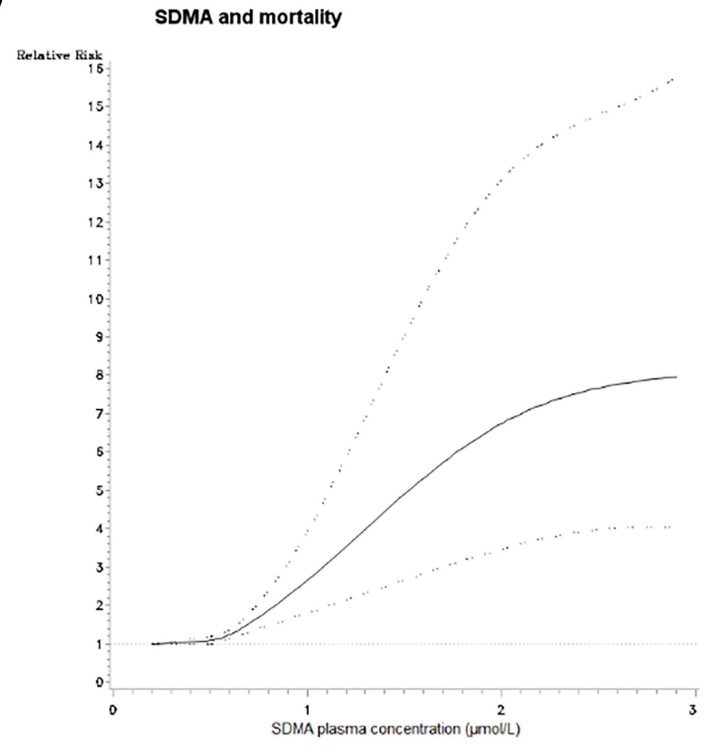

Fig 3. SDMA and all-cause mortality: a) high versus low analysis, and b) non-linear dose-response analysis (based on 4 studies, $\mathbf{p}$ for non-linearity $\mathbf{\mathbf { 0 . 0 1 0 }}$ ). Black square: point estimate for individual study; horizontal line: $95 \% \mathrm{Cl}$ for observed effect in each study; diamond: pooled estimate and $95 \% \mathrm{Cl}$ for meta-analysis. Random-effects estimate (DerSimonian and Laird method).

doi:10.1371/journal.pone.0165811.g003 
Table 3. Meta-Analysis of SDMA and all-cause mortality or CVD for subgroups.

\begin{tabular}{|c|c|c|c|c|c|c|}
\hline Factors stratified & $\begin{array}{c}\text { summary RR } \\
(95 \% \mathrm{Cl}) \neq\end{array}$ & $\begin{array}{c}\text { No. of } \\
\text { studies }\end{array}$ & $\begin{array}{c}\text { P for heterogeneity between } \\
\text { subgroups } t\end{array}$ & $\begin{array}{c}\text { summary RR } \\
(95 \% \mathrm{Cl}) \neq\end{array}$ & $\begin{array}{c}\text { No. of } \\
\text { studies }\end{array}$ & $\begin{array}{c}\text { P for heterogeneity between } \\
\text { subgroups } \dagger\end{array}$ \\
\hline & & All-caus & mortality & & & \\
\hline All & $1.31(1.18-1.46)$ & 17 & & $1.36(1.10-1.68)$ & 13 & \\
\hline Study population & & & & & & \\
\hline General population & $1.71(1.39-2.10)$ & 2 & 0.003 & $2.00(1.42-2.82)$ & 3 & 0.023 \\
\hline $\begin{array}{l}\text { Patients with renal } \\
\text { diseases }\end{array}$ & $1.12(0.99-1.25)$ & 5 & & $1.12(0.88-1.41)$ & 6 & \\
\hline $\begin{array}{l}\text { Patients with existing } \\
\text { CVD }\end{array}$ & $1.52(1.15-2.02)$ & 7 & & $1.27(0.79-2.05)$ & 5 & \\
\hline Patients with diabetes & - & - & & - & - & \\
\hline $\begin{array}{l}\text { Patients from } \\
\text { intensive care unit } \\
\text { S }\end{array}$ & $1.28(0.84-1.94)$ & 4 & & - & & \\
\hline Number of events & & & & & & \\
\hline$<100$ & $1.14(0.92-1.42)$ & 6 & 0.009 & $1.52(1.08-2.16)$ & 9 & 0.583 \\
\hline $100-<200$ & $1.06(0.99-1.13)$ & 7 & & $1.51(1.09-2.09)$ & 2 & \\
\hline$\geq 200$ & $1.52(1.22-1.90)$ & 4 & & $1.17(0.76-1.78)$ & 2 & \\
\hline Duration of follow-up (b & y mean)* & & & & & \\
\hline$<4.7$ years & $1.13(1.02-1.25)$ & 9 & 0.004 & $1.19(0.83-1.70)$ & 6 & 0.297 \\
\hline$\geq 4.7$ years & $1.59(1.29-1.96)$ & 6 & & $1.52(1.14-2.03)$ & 7 & \\
\hline Blood sample & & & & & & \\
\hline Plasma & $1.27(1.11-1.46)$ & 12 & 0.369 & $1.23(0.97-1.56)$ & 10 & 0.134 \\
\hline Serum & $1.46(1.11-1.91)$ & 5 & & $1.56(1.28-1.91)$ & 3 & \\
\hline Method & & & & & & \\
\hline HPLC & $1.18(1.04-1.33)$ & 9 & 0.020 & $1.31(0.98-1.76)$ & 8 & 0.563 \\
\hline $\begin{array}{l}\text { Tandem mass } \\
\text { spectrometry }\end{array}$ & $1.72(1.28-2.30)$ & 7 & & $1.52(1.01-2.29)$ & 5 & \\
\hline Adjustment for importa & nt confounders & & & & & \\
\hline $0-2$ & $2.26(1.28-3.99)$ & 7 & 0.055 & $\begin{array}{l}5.10(0.96- \\
27.15)\end{array}$ & 2 & 0.214 \\
\hline $3-5$ & $1.32(0.97-1.78)$ & 4 & & $1.31(0.57-3.04)$ & 4 & \\
\hline$\geq 6$ & $1.15(1.04-1.27)$ & 6 & & $1.15(0.96-1.37)$ & 7 & \\
\hline
\end{tabular}

$\mathrm{Cl}$, confidence interval; CVD, cardiovascular disease; HPLC, High-performance liquid chromatography; HR, hazard ratio.

₹ summary RRs are derived from the maximally adjusted models.

${ }^{\dagger} \mathrm{P}$ for heterogeneity between subgroups was evaluated by meta-regression analysis.

$\S$ only one study investigated SDMA and all-cause mortality or CVD, respectively in patients with diabetes.

* for all-cause mortality for two studies time of follow-up was not available.

doi:10.1371/journal.pone.0165811.t003 
a)

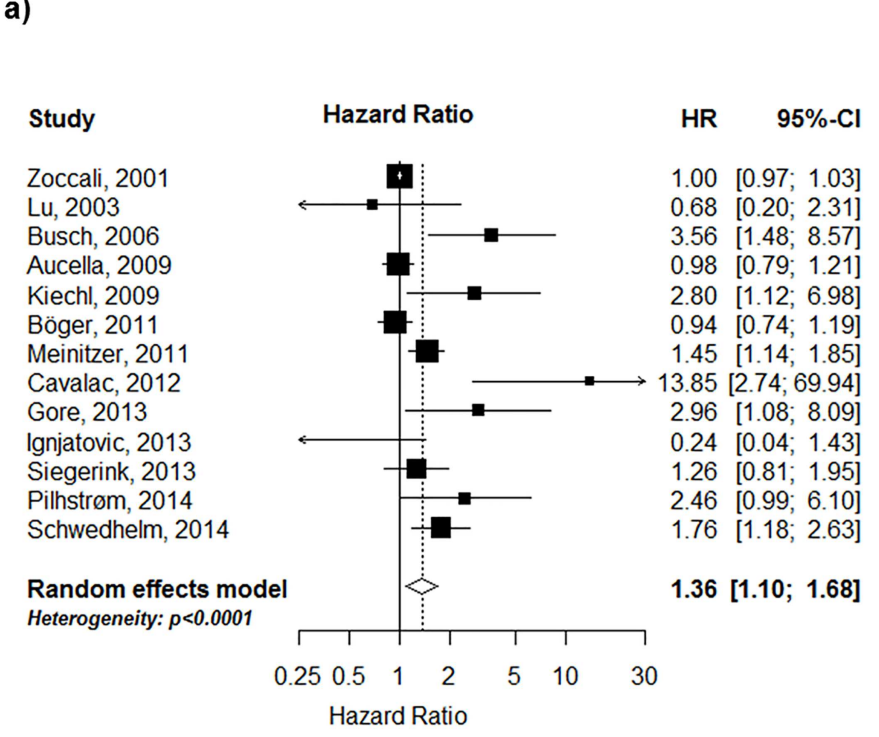

b)

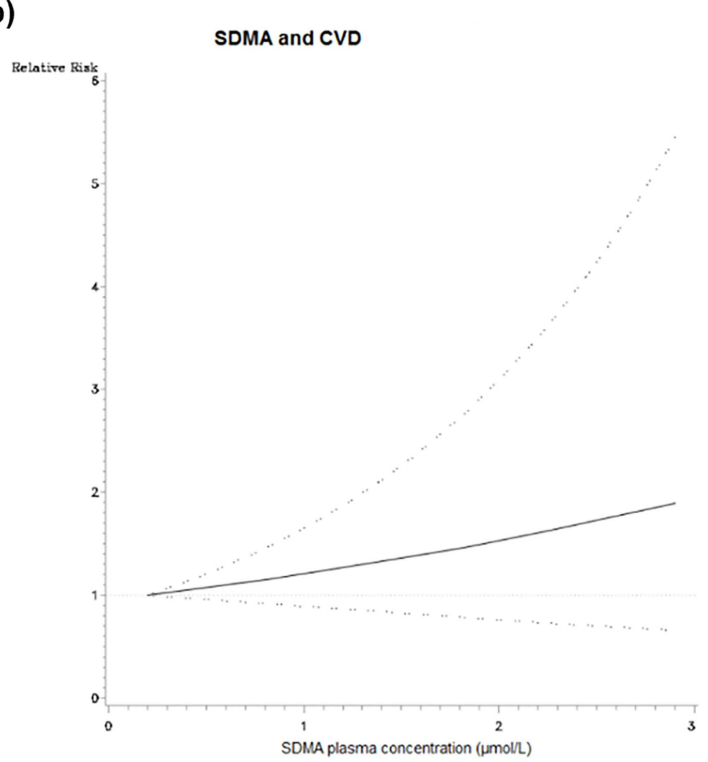

Fig 4. SDMA and CVD: a) high versus low analysis, and b) non-linear dose-response analysis (based on 5 studies, $p$ for nonlinearity $=0.059$ ). Black square: point estimate for individual study; horizontal line: $95 \% \mathrm{Cl}$ for observed effect in each study; diamond: pooled estimate and $95 \% \mathrm{Cl}$ for meta-analysis. Random-effects estimate (DerSimonian and Laird method).

doi:10.1371/journal.pone.0165811.g004

\section{Discussion}

The main results of our systematic review and meta-analysis were as follows. First, circulating concentrations of both biomarkers, ADMA and SDMA, were independently associated with a modestly increased risk of all-cause mortality and of incident CVD. Second, the association of ADMA with CVD was robust in all examined subgroups and across all study populations; its association with mortality was likewise observed in many subgroups but particularly strong in critically ill individuals. Third, the association with SDMA and both outcomes, mortality and CVD, was particularly strong in samples from the general population.

\section{In the context of the published literature}

ADMA and all-cause mortality. Individuals with ADMA levels in the top tertile had an about $50 \%$ increased risk of all-cause mortality compared with individuals in the bottom tertile of ADMA levels and was particularly strong in critically ill patients on intensive care units. In the literature, the most profound elevations of ADMA have been reported in patients with organ failure such as seen in sepsis. Thus the extent of elevation of ADMA in critically ill patients may simply reflect the severity of organ failure,[34] which in turn is strongly related to adverse outcomes. In addition, based on excess mortality observed in clinical trials of NOSinhibitors in critically ill patients, [71] it has been speculated that ADMA may also aggravate clinical outcomes.[72] Recent data from mice with impaired ADMA metabolism point in the same direction.[73]

As expected, slight differences between studies were observed, with slightly smaller effects being reported in studies of higher quality (higher number of events, longer duration of followup and higher number of included confounders in the statistical analysis). In addition, associations were slightly stronger for studies that measured ADMA in plasma compared to studies measuring it in the serum. In our dose-response meta-analysis there was evidence for a non- 
linear relation between ADMA and all-cause mortality, with a steeper increase in risk for ADMA levels around $>0.6 \mu \mathrm{mol} / 1$; however, with ADMA concentrations around $>3.0 \mu \mathrm{mol} / \mathrm{l}$ the risk slightly decreased. In a sensitivity analysis, restricted to studies that assessed ADMA by tandem mass spectrometry and ELISA, the association between ADMA and all-cause mortality was almost linear.

ADMA and CVD. We observed a relative CVD risk increase of $33 \%$ in individuals in the top vs. the bottom ADMA tertile. Our results are consistent with a previous, slightly smaller meta-analysis based on 22 prospective studies, including 19,842 subjects and 2,339 CVD events. Indeed, Willeit and colleagues reported a very similar summary effect estimate [RR (95\%CI): 1.42 (1.29-1.56)]. Exclusion criteria of the latter meta-analysis were stricter compared to our criteria, and thus our meta-analysis was based on more studies and a higher number of individuals and events (30 studies, 30,624 subjects and 3,396 CVD cases). Our results are consistent with some prior analyses in individual studies and we demonstrate that after including all currently available studies we see a positive association between ADMA and CVD, however, some individual studies, including the Framingham study, failed to observe an association between ADMA and CVD.

SDMA and all-cause mortality. Our meta-analysis indicated that individuals in the top SDMA tertile were at higher risk (RR: $31 \%$ ) of dying from any cause as compared to individuals in the bottom SDMA tertile. The association was strongest in samples from the general population and less prominent in patients with prevalent cardiovascular disease. In patients with renal failure, the association failed to reach statistical significance. SDMA is much more sensitive to changes in renal function as compared to ADMA,[74] which may explain its weaker independent association with adverse outcomes in renal disease when renal function is adjusted for. Similar to the findings regarding ADMA and all-cause mortality, the strongest increase of risk was observed at SDMA levels approximately $>0.6 \mu \mathrm{mol} / \mathrm{l}$, and with extreme values, the risk increase was attenuated. However, excluding HPLC-based SDMA measurement, there was no indication for a non-linear association.

SDMA and CVD. In our meta-analysis, SDMA was associated with $36 \%$ (95\% CI: $10 \%$ to $68 \%$ ) increased risk of CVD, by comparing individuals in the top tertile with individuals in the bottom tertile of SDMA. In the meta-analysis mentioned above, a very similar risk estimate was reported that failed to reach statistical significance, [summary RR (95\% CI) 1.32 (0.921.90)], probably due to the smaller number of studies $(n=8$; total $n=9,070)$ and events $(\mathrm{n}=848)$ included.[30] In our report, we included 13 studies with a total of 16,807 individuals (events $=1,534$ ). Our findings were consistent in analyses stratified by study characteristics, method of ADMA assessment and duration of follow-up. However, as in our analyses relating SDMA to all-cause mortality, the association with CVD risk was likewise strongest in samples from the general population.

\section{Change in methods of biomarker determination over time}

Stratification of studies by the methodology used to determine ADMA/SDMA showed some degree of heterogeneity with respect to total mortality which is unlikely to be attributable to clinical differences between the underlying studies.

As with any new biomarker, the methodology for the quantification of ADMA and SDMA has advanced over recent years and has become more and more standardized.[75] Initial clinical studies predominantly relied on high pressure liquid chromatography (HPLC) methods for the detection of ADMA and SDMA.[3, 7, 8] In these early studies, biomarker quantification-especially in absolute terms - was rather challenging due to the limited availability of defined pure ADMA and SDMA standards and reference samples. Limited 
knowledge regarding the prevalence and interference by chemically related compounds added further uncertainties. This may explain the continuous decline in the mean ADMA plasma concentration reported for similar clinical settings, going down from $0.95 \mu \mathrm{mol} / \mathrm{l}$ for healthy controls in 2001 to $0.41 \mu \mathrm{mol} / \mathrm{l}$ in 2011.[7, 76] In the case of SDMA, matters may have been further aggravated by the fact that it was considered to be a biologically inactive byproduct and thus may have received less methodological scrutiny. The less well validated methodology for SDMA determination may also have contributed to the initially mostly negative results regarding SDMA as a risk marker. It may also explain why commercial immunoassays emerged much earlier for ADMA than for SDMA.[9, 32] The introduction of mass spectrometric methods allowed the more reliable determination of reference values for ADMA and SDMA, [77-79] which may help to calibrate and (re)interpret early ADMA and SDMA data.

\section{ADMA and SDMA levels and risk prediction and clinical utility}

In our present-meta analysis, we confirm a positive and statistical significant association of ADMA and SDMA with all-cause mortality and CVD. All associations were relatively consistent across multiple subgroups, but varied in magnitude. In order for ADMA or SDMA measurements to be considered applicable for routine clinical use it needs to be demonstrated, that measuring ADMA or SDMA affects patient management and ultimately patient outcome. $[80,81]$

Wang et al. suggested possible additive effects of ADMA and SDMA with respect to the predictions of major adverse cardiac events and proposed an "arginine methylation index" ([ADMA+SDMA]/Monomethyl-Arginine).[59] This index was statistically independently associated with MACE. However, only few studies investigated whether the addition of ADMA or SDMA improves established indices of risk prediction models, including improvement in discrimination, calibration, and reclassification.[81] Gore and colleagues reported statistically significant increase in the C-statistic after adding SDMA to a model predicting all-cause mortality and CVD in the general population.[15] However, the absolute increments in discrimination were unlikely to be clinically relevant. Other studies did not find a relevant increment in the C-statistic once ADMA or SDMA were added to the statistical prediction models in different clinical settings.[11, 15, 18, 23, 69] This concept also warrants further investigation. It needs to be established, however, whether inclusion of ADMA or SDMA improves risk prediction models (e. g. discrimination, reclassification) [81] beyond classic risk factors or that the increased risk for CVD and mortality associated with higher ADMA or SDMA can be specifically treated. It can be speculated that in clinical practice determination ADMA may be especially useful in critically ill patients while SDMA may be more suitable and useful in the general population. Further studies are warranted to confirm or refute this hypothesis.

In contrast to other risk markers like LDL-cholesterol distinct pharmacological targeting of ADMA or SDMA has not been clinically successful, so far. Still, assuming a causal role in CVD further approaches have been proposed[82]:

1. Direct targeting of circulating ADMA or SDMA. In principle ADMA or SDMA concentrations could be reduced by a decrease of dietary uptake or endogenous generation of ADMA/ SDMA or by increased elimination (i.e. increase of cellular exchange, transport and metabolism).[2]

2. Treatment of putative pathophysiological effects of ADMA or SDMA. In case of ADMA, Larginine has widely been advocated as an "antidote".[83] However, supplementation of Larginine irrespective of underlying ADMA-levels failed to improve cardiovascular outcome 
and prospective studies with long term supplementation of L-arginine in patients with elevation of ADMA and follow-up for relevant clinical endpoints (mortality) remain to be conducted.[84]

\section{Shared and distinct properties of ADMA and SDMA}

ADMA and SDMA are chemically closely related and both associated with incident CVD and total mortality. However, the present study indicates that both compounds may slightly differ in the pattern and in the strength of their association with clinical endpoints within the same cohort as well as across distinct clinical settings.

Methodological aspects, discussed further above, aside these observations are not surprising, considering the overlapping as well as distinct metabolic and (patho-) physiological properties of ADMA and SDMA (summarized in Table 4). It also is possible, if not likely, that ADMA and SDMA may act both as risk markers as well as risk factors. The plasma levels of ADMA and SDMA are affected by several overlapping as well as distinct metabolic pathways. [3, 4, 85-90] Elevation of ADMA may predominantly represent impaired dimethylarginine dimethylaminohydrolase (DDAH) activity [4] while elevation of SDMA more likely reflects impaired renal function [91, 92] and/or impaired alanine-glyoxylate aminotransferase 2 (AGXT2) activity (i.e. hyper beta-aminoisobutyric acid uria).[89, 93, 94] Moreover, ADMA and SDMA have overlapping as well as distinct biological effects (Table 4). While both compounds may alter cellular exchange (i.e. transport) of L-arginine,[1] only ADMA appears to be a direct inhibitor of NOS. $[2,3]$ Independent of the L-arginine-NO-pathway SDMA may exercise biological effects through alternative mechanisms augmenting oxidative stress or activate toll-like receptors. $[95,96]$

\section{Strengths and limitations}

Our meta-analysis has several strengths. To our knowledge, there is no prior large scale metaanalysis quantifying the association between ADMA, SDMA and all-cause mortality. Regarding the association of both biomarkers with CVD, the present study includes a much larger number of studies, patients and clinical events than any previous review or meta-analysis for ADMA or SDMA. This allowed us to consider key methodological factors and subgroup analyses. In addition, we also performed a dose-response meta-analysis of the relation between ADMA; SDMA and all-cause mortality or CVD and assessed the exact shape of the association between biomarker and outcome.

However, our meta-analysis has some limitations that merit consideration. First, we observed high heterogeneity between studies included in our meta-analysis. Thus, we performed stratified analyses by accounting for different study populations, study characteristics and assessment methods of ADMA and SDMA. Slight differences between subgroups were detected, but in general, effects were present across all or most strata. Second, regarding the dose-response analysis, the number of studies we could include was limited because of partially incomplete information in individual studies. Thus, we were not able to stratify the doseresponse meta-analysis by different populations or methodological approaches, and more research exploring dose-response relations is warranted. Third, the majority of the included studies were based on diseased cohorts and evidence of the association between particularly SDMA and all-cause mortality or CVD in the community is still rare. Thus, more populationbased studies investigating this association are needed. Fourth, we conducted our literature search in Medline only and thus, it might be possible that we missed few of the relevant studies. However, we hand-searched all included studies and reviews to check for additional relevant articles. 
Table 4. Differences and similarities in the biological properties of ADMA and SDMA.

\begin{tabular}{|c|c|c|}
\hline & ADMA & SDMA \\
\hline \multirow[t]{2}{*}{$\begin{array}{l}\text { Generation / } \\
\text { source [2] }\end{array}$} & $\begin{array}{l}\text { Endogenous formation [3] } \\
\text { - Monomethylation of protein-bound L- } \\
\text { arginine by type I and II PRMTs [85] } \\
\text { - Asymmetric dimethylation of protein- } \\
\text { bound monomethylarginine by type I } \\
\text { PRMTs [85] } \\
\text { - Liberation of ADMA by protein } \\
\text { degradation [86] }\end{array}$ & $\begin{array}{l}\text { Endogenous formation [3] } \\
\text { - Monomethylation of protein- bound L- } \\
\text { arginine by type I and II PRMTs [85] } \\
\text { - Asymmetric dimethylation of protein-bound } \\
\text { monomethylarginine by type II PRMTs [85] } \\
\text { - Liberation of SDMA by protein degradation } \\
\text { [87] }\end{array}$ \\
\hline & $\frac{\text { Exogenous/ dietary uptake }}{\text { - Exact contribution unknown }}$ & $\frac{\text { Exogenous/ dietary uptake }}{\text { - Exact contribution unknown }}$ \\
\hline $\begin{array}{l}\text { Distribution / } \\
\text { transport [88] }\end{array}$ & $\begin{array}{l}\text { - Cellular uptake and efflux mediated by } \\
\text { cationic amino acid transporters }\end{array}$ & $\begin{array}{l}\text { - Cellular uptake and efflux mediated by } \\
\text { cationic amino acid transporters }\end{array}$ \\
\hline \multirow[t]{2}{*}{ Elimination [2] } & $\begin{array}{l}\text { Metabolism } \\
\text { - Major route of elimination }[3,89,90] \\
\text { - Major metabolising enzymes } \\
\text { 1. DDAH1 and DDAH2 [4] } \\
\text { 2. AGXT2 [91] } \\
\text { 3. Butylation and Methylation [90] }\end{array}$ & $\begin{array}{l}\text { Metabolism } \\
\text { Minor route of elimination }[89,90] \\
\text { 1. No substrate of DDAHs } \\
\text { 2. AGXT2 [91] (Major metabolising } \\
\text { enzyme) } \\
\text { 3. Butylation and Methylation }[90,94]\end{array}$ \\
\hline & $\frac{\text { Renal Excretion }}{\text { - Minor route of elimination }[92,93]}$ & $\frac{\text { Renal Excretion }}{\text { - Major route of elimination }[92,93]}$ \\
\hline $\begin{array}{l}\text { Biological } \\
\text { effects }\end{array}$ & $\begin{array}{l}\text { - Inhibition of nitric oxide synthases } \\
\text { (eNOS, nNOS and iNOS) [2, 3] } \\
\text { - Weak inhibition of L-arginine transport } \\
\text { [88] } \\
\text { - Activation of NF-kB with enhanced } \\
\text { expression of inflammatory cytokines } \\
\text { [95] }\end{array}$ & $\begin{array}{l}\text { - No clinically relevant direct inhibition of } \\
\text { nitric oxide synthases [3] } \\
\text { - Possible weak indirect inhibition of NO- } \\
\text { Synthesis [96] } \\
\text { - Weak inhibition of L-arginine transport [88] } \\
\text { - Activation of NF-kB with enhanced } \\
\text { expression of inflammatory cytokines [97] } \\
\text { - Increase in monocytic ROS production by } \\
\text { enhanced activation of store-operated Ca2 } \\
\text { +- channels [98] } \\
\text { - Modification of HDL activating toll like } \\
\text { Receptors [99] }\end{array}$ \\
\hline
\end{tabular}

ADMA, Asymmetric dimethylarginine; AGXT2; alanine—glyoxylate aminotransferase 2; DDAH, dimethylarginine dimethylaminohydrolase; eNOS, endothelial nitric-oxide synthase; HDL, high density lipoprotein; iNOS, inducible nitric-oxide synthase; NF-kB, nuclear factor kappa-light-chain-enhancer of activated B cells; nNOS; neuronal nitric oxide synthase; PRMT, protein arginine N-methyltransferase; ROS, reactive oxygen species; SDMA, symmetrical dimethylarginine

doi:10.1371/journal.pone.0165811.t004

\section{Conclusions}

In conclusion, this meta-analysis including a large number of prospective studies indicates that ADMA and SDMA are independently associated with all-cause mortality and CVD across a broad spectrum of populations and clinical conditions.

\section{Supporting Information}

S1 Fig. PRISMA checklist.

(PDF) 
S2 Fig. Flowchart of study selection for the meta-analysis.

(PDF)

S1 Table. Considered confounders of each study included in the meta-analysis. (PDF)

\section{Author Contributions}

Conceptualization: WL RM.

Data curation: SS SRS RM.

Formal analysis: SS.

Investigation: SS WL RM.

Methodology: SS WL RM.

Project administration: WL RM.

Resources: WL RM.

Visualization: SS.

Writing - original draft: SS WL RM.

Writing - review \& editing: SS SRS WL RM.

\section{References}

1. Strobel J, Müller F, Zolk O, Endress B, König J, Fromm MF, et al. Transport of asymmetric dimethylarginine (ADMA) by cationic amino acid transporter 2 (CAT2), organic cation transporter 2 (OCT2) and multidrug and toxin extrusion protein 1 (MATE1). Amino Acids. 2013; 45(4):989-1002. Epub 2013/07/ 19. doi: 10.1007/s00726-013-1556-3 PMID: 23864433

2. Kittel A, Maas R. Pharmacology and clinical pharmacology of methylarginines used as inhibitors of nitric oxide synthases. Curr Pharm Des. 2014; 20(22):3530-47. PMID: 24180385.

3. Vallance P, Leone A, Calver A, Collier J, Moncada S. Accumulation of an endogenous inhibitor of nitric oxide synthesis in chronic renal failure. Lancet. 1992; 339(8793):572-5. PMID: 1347093.

4. Leiper J, Nandi M, Torondel B, Murray-Rust J, Malaki M, O'Hara B, et al. Disruption of methylarginine metabolism impairs vascular homeostasis. Nat Med. 2007; 13(2):198-203. doi: 10.1038/nm1543 PMID: 17273169.

5. Jacobi J, Maas R, Cardounel AJ, Arend M, Pope AJ, Cordasic N, et al. Dimethylarginine dimethylaminohydrolase overexpression ameliorates atherosclerosis in apolipoprotein E-deficient mice by lowering asymmetric dimethylarginine. Am J Pathol. 2010; 176(5):2559-70. Epub 2010/03/30. S0002-9440 (10)60051-X [pii] doi: 10.2353/ajpath.2010.090614 PMID: 20348244; PubMed Central PMCID: PMC2861120.

6. Kielstein JT, Impraim B, Simmel S, Bode-Böger SM, Tsikas D, Frölich JC, et al. Cardiovascular effects of systemic nitric oxide synthase inhibition with asymmetrical dimethylarginine in humans. Circulation. 2004; 109(2):172-7. Epub 2003/12/10. doi: 10.1161/01.CIR.0000105764.22626.B1 01. CIR.0000105764.22626.B1 [pii]. PMID: 14662708.

7. Zoccali C, Bode-Böger S, Mallamaci F, Benedetto F, Tripepi G, Malatino L, et al. Plasma concentration of asymmetrical dimethylarginine and mortality in patients with end-stage renal disease: a prospective study. Lancet. 2001; 358(9299):2113-7. Epub 2002/01/11. S0140673601072178 [pii]. PMID: 11784625.

8. Valkonen VP, Paiva H, Salonen JT, Lakka TA, Lehtimaki T, Laakso J, et al. Risk of acute coronary events and serum concentration of asymmetrical dimethylarginine. Lancet. 2001; 358(9299):2127-8. Epub 2002/01/11. S0140-6736(01)07184-7 [pii] doi: 10.1016/S0140-6736(01)07184-7 PMID: 11784629.

9. Schnabel R, Blankenberg S, Lubos E, Lackner KJ, Rupprecht HJ, Espinola-Klein C, et al. Asymmetric dimethylarginine and the risk of cardiovascular events and death in patients with coronary artery 
disease: results from the AtheroGene Study. Circ Res. 2005; 97(5):e53-9. Epub 2005/08/16. 01. RES.0000181286.44222.61 [pii] doi: 10.1161/01.RES.0000181286.44222.61 PMID: 16100045.

10. Böger RH, Endres HG, Schwedhelm E, Darius H, Atzler D, Lüneburg N, et al. Asymmetric dimethylarginine as an independent risk marker for mortality in ambulatory patients with peripheral arterial disease. J Intern Med. 2011; 269(3):349-61. Epub 2010/12/24. doi: 10.1111/j.1365-2796.2010.02322.x PMID: 21175900.

11. Meinitzer A, Kielstein JT, Pilz S, Drechsler C, Ritz E, Boehm BO, et al. Symmetrical and asymmetrical dimethylarginine as predictors for mortality in patients referred for coronary angiography: the Ludwigshafen Risk and Cardiovascular Health study. Clin Chem. 2011; 57(1):112-21. Epub 2010/11/03. clinchem.2010.150854 [pii] doi: 10.1373/clinchem.2010.150854 PMID: 21036946.

12. Böger $R H$, Maas $R$, Schulze $F$, Schwedhelm E. Asymmetric dimethylarginine (ADMA) as a prospective marker of cardiovascular disease and mortality-an update on patient populations with a wide range of cardiovascular risk. Pharmacol Res. 2009; 60(6):481-7. Epub 2009/07/15. S1043-6618(09)00175-3 [pii] doi: 10.1016/j.phrs.2009.07.001 PMID: 19596069.

13. Aucella F, Maas R, Vigilante M, Tripepi G, Schwedhelm E, Margaglione M, et al. Methylarginines and mortality in patients with end stage renal disease: a prospective cohort study. Atherosclerosis. 2009; 207(2):541-5. Epub 2009/06/09. S0021-9150(09)00406-7 [pii] doi: 10.1016/j.atherosclerosis.2009.05. 011 PMID: 19501358.

14. Zeller M, Korandji C, Guilland JC, Sicard P, Vergely C, Lorgis L, et al. Impact of asymmetric dimethylarginine on mortality after acute myocardial infarction. Arterioscler Thromb Vasc Biol. 2008; 28(5):95460. Epub 2008/02/16. ATVBAHA.108.162768 [pii] doi: 10.1161/ATVBAHA.108.162768 PMID: 18276906.

15. Gore MO, Lüneburg N, Schwedhelm E, Ayers CR, Anderssohn M, Khera A, et al. Symmetrical dimethylarginine predicts mortality in the general population: observations from the Dallas heart study. Arterioscler Thromb Vasc Biol. 2013; 33(11):2682-8. Epub 2013/09/07. ATVBAHA.113.301219 [pii] doi: 10.1161/ATVBAHA.113.301219 PMID: 24008162.

16. Schwedhelm E, Wallaschofski H, Atzler D, Dörr M, Nauck M, Völker U, et al. Incidence of all-cause and cardiovascular mortality predicted by symmetric dimethylarginine in the population-based study of health in pomerania. PLoS One. 2014; 9(5):e96875. Epub 2014/05/14. [pii]. PMID: 24819070; PubMed Central PMCID: PMC4018357.

17. Schwedhelm E, Böger $\mathrm{RH}$. The role of asymmetric and symmetric dimethylarginines in renal disease. Nat Rev Nephrol. 2011; 7(5):275-85. Epub 2011/03/30. nrneph.2011.31 [pii] doi: 10.1038/nrneph. 2011.31 PMID: 21445101.

18. Böger RH, Sullivan LM, Schwedhelm E, Wang TJ, Maas R, Benjamin EJ, et al. Plasma asymmetric dimethylarginine and incidence of cardiovascular disease and death in the community. Circulation. 2009; 119(12):1592-600. Epub 2009/03/18. CIRCULATIONAHA.108.838268 [pii] doi: 10.1161/ CIRCULATIONAHA.108.838268 PMID: 19289633; PubMed Central PMCID: PMC2742491.

19. Borgeraas H, Strand E, Ringdal Pedersen E, Dierkes J, Ueland PM, Seifert R, et al. Omega-3 Status and the Relationship between Plasma Asymmetric Dimethylarginine and Risk of Myocardial Infarction in Patients with Suspected Coronary Artery Disease. Cardiol Res Pract. 2012; 2012:201742. Epub 2013/01/25. doi: 10.1155/2012/201742 PMID: 23346455; PubMed Central PMCID: PMC3549394.

20. Siegerink B, Maas R, Vossen CY, Schwedhelm E, Koenig W, Boger R, et al. Asymmetric and symmetric dimethylarginine and risk of secondary cardiovascular disease events and mortality in patients with stable coronary heart disease: the KAROLA follow-up study. Clin Res Cardiol. 2013; 102(3):193-202. Epub 2012/10/18. doi: 10.1007/s00392-012-0515-4 PMID: 23073705.

21. Young JM, Terrin N, Wang X, Greene T, Beck GJ, Kusek JW, et al. Asymmetric dimethylarginine and mortality in stages 3 to 4 chronic kidney disease. Clin J Am Soc Nephrol. 2009; 4(6):1115-20. Epub 2009/04/25. CJN.06671208 [pii] doi: 10.2215/CJN.06671208 PMID: 19389824; PubMed Central PMCID: PMC2689879.

22. Abedini S, Meinitzer A, Holme I, Marz W, Weihrauch G, Fellstrom B, et al. Asymmetrical dimethylarginine is associated with renal and cardiovascular outcomes and all-cause mortality in renal transplant recipients. Kidney Int. 2010; 77(1):44-50. Epub 2009/10/23. ki2009382 [pii] doi: 10.1038/ki.2009.382 PMID: 19847152.

23. Levin A, Rigatto C, Barrett B, Madore F, Muirhead N, Holmes D, et al. Biomarkers of inflammation, fibrosis, cardiac stretch and injury predict death but not renal replacement therapy at 1 year in a Canadian chronic kidney disease cohort. Nephrol Dial Transplant. 2014; 29(5):1037-47. Epub 2013/12/29. gft479 [pii] doi: 10.1093/ndt/gft479 PMID: 24371297.

24. Pihlstrom H, Mjoen G, Dahle DO, Pilz S, Midtvedt K, Marz W, et al. Symmetric dimethylarginine as predictor of graft loss and all-cause mortality in renal transplant recipients. Transplantation. 2014; 98 (11):1219-25. Epub 2014/07/08. doi: 10.1097/TP.0000000000000205 PMID: 24999963; PubMed Central PMCID: PMC4240460. 
25. Lajer M, Tarnow L, Jorsal A, Teerlink T, Parving HH, Rossing P. Plasma concentration of asymmetric dimethylarginine (ADMA) predicts cardiovascular morbidity and mortality in type 1 diabetic patients with diabetic nephropathy. Diabetes Care. 2008; 31(4):747-52. Epub 2007/12/29. dc07-1762 [pii] doi: 10.2337/dc07-1762 PMID: 18162497.

26. Cavusoglu E, Ruwende C, Chopra V, Poludasu S, Yanamadala S, Frishman WH, et al. Relation of baseline plasma ADMA levels to cardiovascular morbidity and mortality at two years in men with diabetes mellitus referred for coronary angiography. Atherosclerosis. 2010; 210(1):226-31. Epub 2009/12/ 01. S0021-9150(09)00908-3 [pii] doi: 10.1016/j.atherosclerosis.2009.10.034 PMID: 19944421.

27. Nijveldt RJ, Teerlink T, Van Der Hoven B, Siroen MP, Kuik DJ, Rauwerda JA, et al. Asymmetrical dimethylarginine (ADMA) in critically ill patients: high plasma ADMA concentration is an independent risk factor of ICU mortality. Clin Nutr. 2003; 22(1):23-30. Epub 2003/01/30. S0261561402906138 [pii]. PMID: 12553946.

28. Davis JS, Darcy CJ, Yeo TW, Jones C, McNeil YR, Stephens DP, et al. Asymmetric dimethylarginine, endothelial nitric oxide bioavailability and mortality in sepsis. PLoS One. 2011; 6(2):e17260. Epub 2011/03/03. doi: 10.1371/journal.pone.0017260 PMID: 21364995; PubMed Central PMCID: PMC3041798.

29. Visser M, Vermeulen MA, Richir MC, Teerlink T, Houdijk AP, Kostense PJ, et al. Imbalance of arginine and asymmetric dimethylarginine is associated with markers of circulatory failure, organ failure and mortality in shock patients. Br J Nutr. 2012; 107(10):1458-65. Epub 2011/12/02. S0007114511004648 [pii] doi: 10.1017/S0007114511004648 PMID: 22129964.

30. Willeit P, Freitag DF, Laukkanen JA, Chowdhury S, Gobin R, Mayr M, et al. Asymmetric dimethylarginine and cardiovascular risk: systematic review and meta-analysis of 22 prospective studies. J Am Heart Assoc. 2015; 4(6):e001833. Epub 2015/05/30. JAHA.115.001833 [pii] doi: 10.1161/JAHA.115. 001833 PMID: 26021436; PubMed Central PMCID: PMC4599532.

31. Moher D, Liberati A, Tetzlaff J, Altman DG. Preferred reporting items for systematic reviews and metaanalyses: the PRISMA statement. J Clin Epidemiol. 2009; 62(10):1006-12. Epub 2009/07/28. S08954356(09)00179-6 [pii] doi: 10.1016/j.jclinepi.2009.06.005 PMID: 19631508.

32. Koch A, Weiskirchen R, Bruensing J, Dückers H, Buendgens L, Kunze J, et al. Regulation and prognostic relevance of symmetric dimethylarginine serum concentrations in critical illness and sepsis. Mediators Inflamm. 2013; 2013:413826. Epub 2013/08/13. doi: 10.1155/2013/413826 PMID: 23935249; PubMed Central PMCID: PMC3712234.

33. Ari H, Ari S, Erdogan E, Tiryakioglu O, Huysal K, Koca V, et al. The effects of endothelial dysfunction and inflammation on slow coronary flow. Turk Kardiyol Dern Ars. 2010; 38(5):327-33. Epub 2011/01/ 05. PMID: 21200102.

34. Koch A, Weiskirchen R, Kunze J, Dückers H, Bruensing J, Buendgens L, et al. Elevated asymmetric dimethylarginine levels predict short- and long-term mortality risk in critically ill patients. J Crit Care. 2013; 28(6):947-53. Epub 2013/08/21. S0883-9441(13)00141-X [pii] doi: 10.1016/j.jcrc.2013.05.016 PMID: 23953490.

35. Kodama S, Saito K, Tanaka S, Maki M, Yachi Y, Asumi M, et al. Cardiorespiratory fitness as a quantitative predictor of all-cause mortality and cardiovascular events in healthy men and women: a meta-analysis. JAMA. 2009; 301(19):2024-35. Epub 2009/05/21. 301/19/2024 [pii] doi: 10.1001/jama.2009.681 PMID: 19454641.

36. Skoro-Sajer N, Mittermayer F, Panzenboeck A, Bonderman D, Sadushi R, Hitsch R, et al. Asymmetric dimethylarginine is increased in chronic thromboembolic pulmonary hypertension. Am J Respir Crit Care Med. 2007; 176(11):1154-60. Epub 2007/09/18. 200702-278OC [pii] doi: 10.1164/rccm.2007022780C PMID: 17872491.

37. Anderssohn M, Rosenberg M, Schwedhelm E, Zugck C, Lutz M, Luneburg N, et al. The L-Arginineasymmetric dimethylarginine ratio is an independent predictor of mortality in dilated cardiomyopathy. $J$ Card Fail. 2012; 18(12):904-11. Epub 2012/12/05. S1071-9164(12)01288-2 [pii] doi: 10.1016/j. cardfail.2012.10.011 PMID: 23207078.

38. Plicner D, Mazur P, Sadowski J, Undas A. Asymmetric dimethylarginine and oxidative stress following coronary artery bypass grafting: associations with postoperative outcome. Eur J Cardiothorac Surg. 2014; 45(5):e136-41. Epub 2014/02/14. ezt646 [pii] doi: 10.1093/ejcts/ezt646 PMID: 24523493.

39. Danesh J, Collins R, Appleby P, Peto R. Association of fibrinogen, C-reactive protein, albumin, or leukocyte count with coronary heart disease: meta-analyses of prospective studies. JAMA. 1998; 279 (18):1477-82. Epub 1998/05/26. jma80001 [pii]. PMID: 9600484.

40. Orsini N, Li R, Wolk A, Khudyakov P, Spiegelman D. Meta-analysis for linear and nonlinear doseresponse relations: examples, an evaluation of approximations, and software. Am J Epidemiol. 2012; 175(1):66-73. doi: 10.1093/aje/kwr265 PMID: 22135359; PubMed Central PMCID: PMCPMC3244608. 
41. Greenland S, Longnecker MP. Methods for trend estimation from summarized dose-response data, with applications to meta-analysis. Am J Epidemiol. 1992; 135(11):1301-9. PMID: 1626547.

42. Chene G, Thompson SG. Methods for summarizing the risk associations of quantitative variables in epidemiologic studies in a consistent form. Am J Epidemiol. 1996; 144(6):610-21. Epub 1996/09/15. PMID: 8797521.

43. Aune D, Greenwood DC, Chan DS, Vieira R, Vieira AR, Navarro Rosenblatt DA, et al. Body mass index, abdominal fatness and pancreatic cancer risk: a systematic review and non-linear doseresponse meta-analysis of prospective studies. Ann Oncol. 2012; 23(4):843-52. Epub 2011/09/06. mdr398 [pii] doi: 10.1093/annonc/mdr398 PMID: 21890910.

44. Drew DA, Tighiouart $\mathrm{H}$, Scott $\mathrm{T}$, Kantor A, Fan L, Artusi $\mathrm{C}$, et al. Asymmetric dimethylarginine, race, and mortality in hemodialysis patients. Clin J Am Soc Nephrol. 2014; 9(8):1426-33. Epub 2014/06/28. CJN.00770114 [pii] doi: 10.2215/CJN.00770114 PMID: 24970872; PubMed Central PMCID: PMC4123389.

45. Mittermayer F, Krzyzanowska K, Exner M, Mlekusch W, Amighi J, Sabeti S, et al. Asymmetric dimethylarginine predicts major adverse cardiovascular events in patients with advanced peripheral artery disease. Arterioscler Thromb Vasc Biol. 2006; 26(11):2536-40. Epub 2006/08/26. 01. ATV.0000242801.38419.48 [pii] doi: 10.1161/01.ATV.0000242801.38419.48 PMID: 16931791.

46. Schulze F, Carter AM, Schwedhelm E, Ajjan R, Maas R, von Holten RA, et al. Symmetric dimethylarginine predicts all-cause mortality following ischemic stroke. Atherosclerosis. 2010; 208(2):518-23. Epub 2009/08/25. S0021-9150(09)00599-1 [pii] doi: 10.1016/j.atherosclerosis.2009.06.039 PMID: 19700158.

47. Aune D, Chan DS, Lau R, Vieira R, Greenwood DC, Kampman E, et al. Dietary fibre, whole grains, and risk of colorectal cancer: systematic review and dose-response meta-analysis of prospective studies. BMJ. 2011; 343:d6617. Epub 2011/11/15. PMID: 22074852; PubMed Central PMCID: PMC3213242. doi: $10.1136 / \mathrm{bmj} . \mathrm{d} 6617$

48. Ben $Q$, Sun $Y$, Chai R, Qian A, Xu B, Yuan Y. Dietary fiber intake reduces risk for colorectal adenoma: a meta-analysis. Gastroenterology. 2014; 146(3):689-99 e6. doi: 10.1053/j.gastro.2013.11.003 PMID: 24216326.

49. Durrleman S, Simon R. Flexible regression models with cubic splines. Stat Med. 1989; 8(5):551-61. PMID: 2657958.

50. Lu TM, Ding YA, Lin SJ, Lee WS, Tai HC. Plasma levels of asymmetrical dimethylarginine and adverse cardiovascular events after percutaneous coronary intervention. Eur Heart J. 2003; 24(21):1912-9. Epub 2003/10/31. S0195668X03005694 [pii]. PMID: 14585249.

51. Ravani P, Tripepi G, Malberti F, Testa S, Mallamaci F, Zoccali C. Asymmetrical dimethylarginine predicts progression to dialysis and death in patients with chronic kidney disease: a competing risks modeling approach. J Am Soc Nephrol. 2005; 16(8):2449-55. Epub 2005/06/10. ASN.2005010076 [pii] doi: 10.1681/ASN.2005010076 PMID: 15944335.

52. Busch M, Fleck C, Wolf G, Stein G. Asymmetrical (ADMA) and symmetrical dimethylarginine (SDMA) as potential risk factors for cardiovascular and renal outcome in chronic kidney disease-possible candidates for paradoxical epidemiology? Amino Acids. 2006; 30(3):225-32. Epub 2006/05/09. doi: 10. 1007/s00726-005-0268-8 PMID: 16680555.

53. Maas R, Schulze F, Baumert J, Löwel H, Hamraz K, Schwedhelm E, et al. Asymmetric dimethylarginine, smoking, and risk of coronary heart disease in apparently healthy men: prospective analysis from the population-based Monitoring of Trends and Determinants in Cardiovascular Disease/Kooperative Gesundheitsforschung in der Region Augsburg study and experimental data. Clin Chem. 2007; 53 (4):693-701. Epub 2007/02/24. clinchem.2006.081893 [pii] doi: 10.1373/clinchem.2006.081893 PMID: 17317881.

54. Nicholls SJ, Wang Z, Koeth R, Levison B, DelFraino B, Dzavik V, et al. Metabolic profiling of arginine and nitric oxide pathways predicts hemodynamic abnormalities and mortality in patients with cardiogenic shock after acute myocardial infarction. Circulation. 2007; 116(20):2315-24. Epub 2007/ 10/31. CIRCULATIONAHA.107.693986 [pii] doi: 10.1161/CIRCULATIONAHA.107.693986 PMID: 17967979.

55. Leong T, Zylberstein D, Graham I, Lissner L, Ward D, Fogarty J, et al. Asymmetric dimethylarginine independently predicts fatal and nonfatal myocardial infarction and stroke in women: 24-year follow-up of the population study of women in Gothenburg. Arterioscler Thromb Vasc Biol. 2008; 28(5):961-7. Epub 2008/02/23. ATVBAHA.107.156596 [pii] doi: 10.1161/ATVBAHA.107.156596 PMID: 18292394.

56. Wilson Tang WH, Tong W, Shrestha K, Wang Z, Levison BS, Delfraino B, et al. Differential effects of arginine methylation on diastolic dysfunction and disease progression in patients with chronic systolic heart failure. Eur Heart J. 2008; 29(20):2506-13. Epub 2008/08/09. ehn360 [pii] doi: 10.1093/ eurheartj/ehn360 PMID: 18687662; PubMed Central PMCID: PMC2567021. 
57. Cavusoglu E, Ruwende C, Chopra V, Yanamadala S, Eng C, Pinsky DJ, et al. Relationship of baseline plasma ADMA levels to cardiovascular outcomes at 2 years in men with acute coronary syndrome referred for coronary angiography. Coron Artery Dis. 2009; 20(2):112-7. Epub 2009/02/26. doi: 10. 1097/MCA.0b013e328323982f PMID: 19240644.

58. Kiechl S, Lee T, Santer P, Thompson G, Tsimikas S, Egger G, et al. Asymmetric and symmetric dimethylarginines are of similar predictive value for cardiovascular risk in the general population. Atherosclerosis. 2009; 205(1):261-5. Epub 2008/12/23. S0021-9150(08)00775-2 [pii] doi: 10.1016/j. atherosclerosis.2008.10.040 PMID: 19100547.

59. Wang Z, Tang WH, Cho L, Brennan DM, Hazen SL. Targeted metabolomic evaluation of arginine methylation and cardiovascular risks: potential mechanisms beyond nitric oxide synthase inhibition. Arterioscler Thromb Vasc Biol. 2009; 29(9):1383-91. Epub 2009/06/23. ATVBAHA.109.185645 [pii] doi: 10.1161/ATVBAHA.109.185645 PMID: 19542023; PubMed Central PMCID: PMC2755199.

60. Ari H, Ari S, Erdogan E, Tiryakioglu O, Ustundag Y, Huysal K, et al. A novel predictor of restenosis and adverse cardiac events: asymmetric dimethylarginine. Heart Vessels. 2010; 25(1):19-26. Epub 2010/ 01/22. doi: 10.1007/s00380-009-1158-x PMID: 20091394.

61. Shi B, Ni Z, Zhou W, Yu Z, Gu L, Mou S, et al. Circulating levels of asymmetric dimethylarginine are an independent risk factor for left ventricular hypertrophy and predict cardiovascular events in pre-dialysis patients with chronic kidney disease. Eur J Intern Med. 2010; 21(5):444-8. Epub 2010/09/08. S09536205(10)00141-X [pii] doi: 10.1016/j.ejim.2010.07.001 PMID: 20816602.

62. Yeo TW, Lampah DA, Tjitra E, Gitawati R, Darcy CJ, Jones C, et al. Increased asymmetric dimethylarginine in severe falciparum malaria: association with impaired nitric oxide bioavailability and fatal outcome. PLoS Pathog. 2010; 6(4):e1000868. Epub 2010/04/28. doi: 10.1371/journal.ppat.1000868 PMID: 20421938; PubMed Central PMCID: PMC2858698.

63. Lu TM, Chung MY, Lin MW, Hsu CP, Lin SJ. Plasma asymmetric dimethylarginine predicts death and major adverse cardiovascular events in individuals referred for coronary angiography. Int $\mathrm{J}$ Cardiol. 2011; 153(2):135-40. Epub 2011/07/29. S0167-5273(11)00677-2 [pii] doi: 10.1016/j.ijcard.2011.06. 120 PMID: 21794936.

64. Tripepi G, Mattace Raso F, Sijbrands E, Seck MS, Maas R, Boger R, et al. Inflammation and asymmetric dimethylarginine for predicting death and cardiovascular events in ESRD patients. Clin J Am Soc Nephrol. 2011; 6(7):1714-21. Epub 2011/06/07. CJN.11291210 [pii] doi: 10.2215/CJN.11291210 PMID: 21642364.

65. Cavalca V, Veglia F, Squellerio I, De Metrio M, Rubino M, Porro B, et al. Circulating levels of dimethylarginines, chronic kidney disease and long-term clinical outcome in non-ST-elevation myocardial infarction. PLoS One. 2012; 7(11):e48499. Epub 2012/11/28. doi: 10.1371/journal.pone.0048499 PONE-D-12-17620 [pii]. PMID: 23185262; PubMed Central PMCID: PMC3501498.

66. Hsu CP, Lin SJ, Chung MY, Lu TM. Asymmetric dimethylarginine predicts clinical outcomes in ischemic chronic heart failure. Atherosclerosis. 2012; 225(2):504-10. Epub 2012/10/25. S0021-9150(12) 00658-2 [pii] doi: 10.1016/j.atherosclerosis.2012.09.040 PMID: 23092827.

67. Zairis MN, Patsourakos NG, Tsiaousis GZ, Theodossis Georgilas A, Melidonis A, Makrygiannis SS, et al. Plasma asymmetric dimethylarginine and mortality in patients with acute decompensation of chronic heart failure. Heart. 2012; 98(11):860-4. Epub 2012/03/17. heartjnl-2011-301372 [pii] doi: 10. 1136/heartjnl-2011-301372 PMID: 22422591.

68. Ignjatovic AM, Cvetkovic TP, Pavlovic RM, Dordevic VM, Milosevic ZG, Dordevic VB, et al. Endothelial dysfunction, inflammation and malnutrition markers as predictors of mortality in dialysis patients: multimarker approach. Int Urol Nephrol. 2013; 45(6):1715-24. Epub 2013/04/18. doi: 10.1007/s11255-0130439-6 PMID: 23591722.

69. Pizzarelli F, Maas R, Dattolo P, Tripepi G, Michelassi S, D’Arrigo G, et al. Asymmetric dimethylarginine predicts survival in the elderly. Age (Dordr). 2013; 35(6):2465-75. Epub 2013/04/16. doi: 10.1007/ s11357-013-9523-1 PMID: 23584888; PubMed Central PMCID: PMC3824988.

70. Yilmaz MI, Demirkaya E, Acikel C, Saldir M, Akar S, Cayci T, et al. Endothelial function in patients with familial Mediterranean fever-related amyloidosis and association with cardiovascular events. Rheumatology (Oxford). 2014; 53(11):2002-8. Epub 2014/06/08. keu231 [pii] doi: 10.1093/rheumatology/ keu231 PMID: 24907154.

71. Lopez A, Lorente JA, Steingrub J, Bakker J, McLuckie A, Willatts S, et al. Multiple-center, randomized, placebo-controlled, double-blind study of the nitric oxide synthase inhibitor 546C88: effect on survival in patients with septic shock. Crit Care Med. 2004; 32(1):21-30. Epub 2004/01/07. doi: 10.1097/01. CCM.0000105581.01815.C6 PMID: 14707556.

72. Maas R, Dentz L, Schwedhelm E, Thoms W, Kuss O, Hiltmeyer N, et al. Elevated plasma concentrations of the endogenous nitric oxide synthase inhibitor asymmetric dimethylarginine predict adverse events in patients undergoing noncardiac surgery. Crit Care Med. 2007; 35(8):1876-81. Epub 2007/ 06/22. doi: 10.1097/01.CCM.0000277038.11630.71 PMID: 17581491. 
73. Lambden S, Kelly P, Ahmetaj-Shala B, Wang Z, Lee B, Nandi M, et al. Dimethylarginine dimethylaminohydrolase 2 regulates nitric oxide synthesis and hemodynamics and determines outcome in polymicrobial sepsis. Arterioscler Thromb Vasc Biol. 2015; 35(6):1382-92. Epub 2015/04/11. ATVBAHA.115.305278 [pii] doi: 10.1161/ATVBAHA.115.305278 PMID: 25857313.

74. Kielstein JT, Salpeter SR, Bode-Böger SM, Cooke JP, Fliser D. Symmetric dimethylarginine (SDMA) as endogenous marker of renal function-a meta-analysis. Nephrol Dial Transplant. 2006; 21 (9):2446-51. Epub 2006/06/13. gfl292 [pii] doi: 10.1093/ndt/gfl292 PMID: 16766542.

75. Schwedhelm E. Quantification of ADMA: analytical approaches. Vasc Med. 2005; 10 Suppl 1:S89-95. Epub 2006/02/01. PMID: 16444874.

76. Lüneburg N, Xanthakis V, Schwedhelm E, Sullivan LM, Maas R, Anderssohn M, et al. Reference intervals for plasma $\mathrm{L}$-arginine and the $\mathrm{L}$-arginine:asymmetric dimethylarginine ratio in the Framingham Offspring Cohort. J Nutr. 2011; 141(12):2186-90. Epub 2011/10/28. jn.111.148197 [pii] doi: 10.3945/ jn.111.148197 PMID: 22031661; PubMed Central PMCID: PMC3223876.

77. Atzler D, Schwedhelm E, Nauck M, Ittermann T, Böger RH, Friedrich N. Serum reference intervals of homoarginine, ADMA, and SDMA in the study of health in Pomerania. Clin Chem Lab Med. 2014; 52 (12):1835-42. Epub 2014/06/20. doi: 10.1515/cclm-2014-0314/j/cclm.ahead-of-print/cclm-20140314/cclm-2014-0314.xml [pii]. PMID: 24945429.

78. Schwedhelm E, Xanthakis V, Maas R, Sullivan LM, Schulze F, Riederer U, et al. Asymmetric dimethylarginine reference intervals determined with liquid chromatography-tandem mass spectrometry: results from the Framingham offspring cohort. Clin Chem. 2009; 55(8):1539-45. Epub 2009/06/23. clinchem.2009.124263 [pii] doi: 10.1373/clinchem.2009.124263 PMID: 19541865; PubMed Central PMCID: PMC3794429.

79. Schwedhelm E, Xanthakis V, Maas R, Sullivan LM, Atzler D, Luneburg N, et al. Plasma symmetric dimethylarginine reference limits from the Framingham offspring cohort. Clin Chem Lab Med. 2011; 49 (11):1907-10. Epub 2011/08/26. doi: 10.1515/CCLM.2011.679 PMID: 21864208; PubMed Central PMCID: PMC3235736.

80. Vasan RS. Biomarkers of cardiovascular disease: molecular basis and practical considerations. Circulation. 2006; 113(19):2335-62. Epub 2006/05/17. 113/19/2335 [pii] doi: 10.1161/CIRCULATIONAHA. 104.482570 PMID: 16702488.

81. Hlatky MA, Greenland P, Arnett DK, Ballantyne CM, Criqui MH, Elkind MS, et al. Criteria for evaluation of novel markers of cardiovascular risk: a scientific statement from the American Heart Association. Circulation. 2009; 119(17):2408-16. Epub 2009/04/15. CIRCULATIONAHA.109.192278 [pii] doi: 10.1161/CIRCULATIONAHA.109.192278 PMID: 19364974; PubMed Central PMCID: PMC2956982.

82. Maas R. Pharmacotherapies and their influence on asymmetric dimethylargine (ADMA). Vasc Med. 2005; 10 Suppl 1:S49-57. Epub 2006/02/01. PMID: 16444869.

83. Böger RH. The pharmacodynamics of L-arginine. J Nutr. 2007; 137(6 Suppl 2):1650S-5S. Epub 2007/ 05/22. 137/6/1650S [pii]. PMID: 17513442.

84. Schulman SP, Becker LC, Kass DA, Champion HC, Terrin ML, Forman S, et al. L-arginine therapy in acute myocardial infarction: the Vascular Interaction With Age in Myocardial Infarction (VINTAGE MI) randomized clinical trial. JAMA. 2006; 295(1):58-64. Epub 2006/01/05. 295/1/58 [pii] doi: 10.1001/ jama.295.1.58 PMID: 16391217.

85. Morales $\mathrm{Y}$, Caceres T, May K, Hevel JM. Biochemistry and regulation of the protein arginine methyltransferases (PRMTs). Arch Biochem Biophys. 2016; 590:138-52. doi: 10.1016/j.abb.2015.11.030 PMID: 26612103.

86. Maas R, Tan-Andreesen J, Schwedhelm E, Schulze F, Boger RH. A stable-isotope based technique for the determination of dimethylarginine dimethylaminohydrolase (DDAH) activity in mouse tissue. $J$ Chromatogr B Analyt Technol Biomed Life Sci. 2007; 851(1-2):220-8. doi: 10.1016/j.jchromb.2007. 01.020 PMID: 17296339

87. Shirakawa T, Kako K, Shimada T, Nagashima Y, Nakamura A, Ishida J, et al. Production of free methylarginines via the proteasome and autophagy pathways in cultured cells. Mol Med Rep. 2011; 4 (4):615-20. doi: 10.3892/mmr.2011.488 PMID: 21584492.

88. Strobel J, Mieth M, Endress B, Auge D, König J, Fromm MF, et al. Interaction of the cardiovascular risk marker asymmetric dimethylarginine (ADMA) with the human cationic amino acid transporter 1 (CAT1). J Mol Cell Cardiol. 2012; 53(3):392-400. Epub 2012/06/19. S0022-2828(12)00214-3 [pii] doi: 10.1016/j.yjmcc.2012.06.002 PMID: 22705145.

89. McDermott JR. Studies on the catabolism of $\mathrm{Ng}$-methylarginine, $\mathrm{Ng}, \mathrm{Ng}$-dimethylarginine and $\mathrm{Ng}, \mathrm{Ng}$ dimethylarginine in the rabbit. Biochem J. 1976; 154(1):179-84. PMID: 1275907; PubMed Central PMCID: PMCPMC1172690. 
90. Ogawa T, Kimoto M, Watanabe H, Sasaoka K. Metabolism of NG,NG-and NG,N'G-dimethylarginine in rats. Arch Biochem Biophys. 1987; 252(2):526-37. PMID: 3101600.

91. Caplin B, Wang Z, Slaviero A, Tomlinson J, Dowsett L, Delahaye M, et al. Alanine-glyoxylate aminotransferase-2 metabolizes endogenous methylarginines, regulates NO, and controls blood pressure. Arterioscler Thromb Vasc Biol. 2012; 32(12):2892-900. doi: 10.1161/ATVBAHA.112.254078 PMID: 23023372.

92. Ronden RA, Houben AJ, Teerlink T, Bakker JA, Bierau J, Stehouwer CD, et al. Reduced renal plasma clearance does not explain increased plasma asymmetric dimethylarginine in hypertensive subjects with mild to moderate renal insufficiency. Am J Physiol Renal Physiol. 2012; 303(1):F149-56. doi: 10. 1152/ajprenal.00045.2012 PMID: 22552932.

93. Carello KA, Whitesall SE, Lloyd MC, Billecke SS, D'Alecy LG. Asymmetrical dimethylarginine plasma clearance persists after acute total nephrectomy in rats. Am J Physiol Heart Circ Physiol. 2006; 290(1): H209-16. doi: 10.1152/ajpheart.00208.2005 PMID: 16113067.

94. Martens-Lobenhoffer J, Bode-Boger SM. Amino acid N-acetylation: metabolic elimination of symmetric dimethylarginine as symmetric $\mathrm{N}$ (alpha)-acetyldimethylarginine, determined in human plasma and urine by LC-MS/MS. J Chromatogr B Analyt Technol Biomed Life Sci. 2015; 975:59-64. doi: 10.1016/j. jchromb.2014.11.009 PMID: 25438244.

95. Jiang JL, Wang S, Li NS, Zhang XH, Deng HW, Li YJ. The inhibitory effect of simvastatin on the ADMA-induced inflammatory reaction is mediated by MAPK pathways in endothelial cells. Biochem Cell Biol. 2007; 85(1):66-77. doi: 10.1139/o06-146 PMID: 17464346.

96. Bode-Boger SM, Scalera F, Kielstein JT, Martens-Lobenhoffer J, Breithardt G, Fobker M, et al. Symmetrical dimethylarginine: a new combined parameter for renal function and extent of coronary artery disease. J Am Soc Nephrol. 2006; 17(4):1128-34. doi: 10.1681/ASN.2005101119 PMID: 16481412.

97. Schepers E, Barreto DV, Liabeuf S, Glorieux G, Eloot S, Barreto FC, et al. Symmetric dimethylarginine as a proinflammatory agent in chronic kidney disease. Clin J Am Soc Nephrol. 2011; 6(10):2374-83. doi: 10.2215/CJN.01720211 PMID: 21817129; PubMed Central PMCID: PMCPMC3359555.

98. Schepers E, Glorieux G, Dhondt A, Leybaert L, Vanholder R. Role of symmetric dimethylarginine in vascular damage by increasing ROS via store-operated calcium influx in monocytes. Nephrol Dial Transplant. 2009; 24(5):1429-35. doi: 10.1093/ndt/gfn670 PMID: 19059932.

99. Speer T, Rohrer L, Blyszczuk P, Shroff R, Kuschnerus K, Krankel N, et al. Abnormal high-density lipoprotein induces endothelial dysfunction via activation of Toll-like receptor-2. Immunity. 2013; 38 (4):754-68. doi: 10.1016/j.immuni.2013.02.009 PMID: 23477738. 